\title{
L'espace symétrique de Drinfeld et correspondance de Langlands locale I
}

\author{
Haoran Wang
}

\begin{abstract}
Résumé
Soit $K$ une extension finie de $\mathbb{Q}_{p}$. On étudie la géométrie et la cohomologie du revêtement modéré de l'espace symétrique de Drinfeld sur $K$. On prouve, de manière purement locale, que la cohomologie de degré médian réalise la correspondance de Langlands locale et la correspondance de Jacquet-Langlands locale pour les représentations supercuspidales de niveau zéro.
\end{abstract}

\begin{abstract}
Let $K$ be a finite extension of $\mathbb{Q}_{p}$. We study the geometry and cohomology of the tamely ramified cover of Drinfeld's symmetric space over $K$. We prove, in a purely local way, that the cohomology in middle degree realises the local Langlands correspondence and local JacquetLanglands correspondence for the depth zero supercuspidal representations.
\end{abstract}

\section{Table des matières}

$\begin{array}{llc}1 & \text { Introduction } & 1\end{array}$

2 Sur le revêtement modéré de l'espace symétrique de Drinfeld 3

2.1 Rappels sur l'espace symétrique de Drinfeld . . . . . . . . . . . . . . . 3

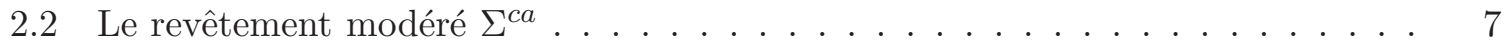

2.3 Le prolongement de $\Sigma^{c a}$ au-dessus d'un sommet . . . . . . . . . . . . . . . 10

2.4 Un calcul du torseur $\bar{\Sigma}_{s}^{0} \ldots \ldots \ldots \ldots \ldots \ldots \ldots \ldots$

2.5 Le lien avec les variétés de Deligne-Lusztig . . . . . . . . . . . . . 17

$\begin{array}{lll}3 & \text { La partie supercuspidale de la cohomologie } & \mathbf{2 1}\end{array}$

3.1 La démonstration de Théorème A . . . . . . . . . . . . . . . . . . 21

$\begin{array}{ll}\text { Références } & 27\end{array}$

\section{Introduction}

Soit $K$ un corps local de caractéristique résiduelle $p$, d'anneau des entiers $\mathcal{O}$ et de corps résiduel $\mathbb{F}_{q}$. Fixons une clôture algébrique $K^{c a}$ de $K$ et un entier $d \geqslant 2$. On dispose de trois groupes $G:=\mathrm{GL}_{d}(K), D^{\times}$les éléments inversibles dans l'algèbre à division $D$ qui est centrale sur $K$ et est d'invariant $1 / d$, et $W_{K}$ le groupe de Weil de $K$. 
Dans [Dri74], Drinfeld a introduit l' "espace symétrique $p$-adique" de dimension $d-1, \Omega^{d-1}$, défini comme le complémentaire de tous les hyperplans $K$-rationels dans l'espace projectif $\mathbb{P}_{K}^{d-1}$. Cet espace est un espace rigide-analytique muni d'une action continue de $G$. Peu après, il a découvert, dans [Dri76], un système projectif de revêtements étales $\left\{\Sigma_{n}\right\}$ (la tour de Drinfeld) de $\Omega^{d-1}$ munis des actions de $G \times D^{\times} \times W_{K}$. Drinfeld et Carayol [Car90] ont conjecturé que la limite inductive de la cohomologie de $\Sigma_{n}$ se décompose en une somme directe sur les séries discrètes $\pi$ de $G$, de $\pi \otimes \mathrm{LJ}(\pi) \otimes \sigma(\pi)$, où $\mathrm{LJ}(\pi)$ désigne la représentation de $D^{\times}$associée à $\pi$ par la correspondance de Jacquet-Langlands, et $\sigma(\pi)$ est l'unique quotient irréductible de la représentation de Weil-Deligne $L(\pi)$ associée à $\pi$ par la correspondance de Langlands normalisée à la Hecke. Lorsque $\pi$ est supercuspidale, $\sigma(\pi)$ coïncide avec $L(\pi)$.

La partie supercuspidale de cette conjecture ainsi que l'énoncé analogue concernant la tour de Lubin-Tate $\left\{\mathcal{M}_{L T, n}\right\}_{n \in \mathbb{N}}$ (la conjecture de Deligne-Carayol) ont été explorés dans les quatres articles [Har97] [Boy99] [HT01] et [Hau05], en caractéristique nulle ou en égales caractéristique, par voie globale en utilisant les variétés de Shimura ou de Drinfeld.

Comme la théorie de Lubin-Tate classique, il est naturel de chercher une preuve de voie locale et plus explicite de ces résultats. L'objet de cet article est d'étudier par voie locale la partie supercuspidale de la cohomologie de $\Sigma_{1}$ (par abus de notation, on le note $\Sigma$ dans les sections qui suivent) lorsque $K$ est de caractéristique nulle (cette étude est aussi valable pour $K$ d'égale caractéristique). La partie non-supercuspidale sera l'objet de [Wan14]. Pour énoncer nos résultats, on utilise une variante de $\Sigma_{1}$ notée $\mathcal{M}_{D r, 1} / \varpi^{\mathbb{Z}}$ qui est une réunion disjointe de $d$-copies de $\Sigma_{1}$. Le théorème principal est le suivant :

Théorème A. (Théorème (3.1.6)) Soit $\rho$ une $\overline{\mathbb{Q}}_{\ell}$-représentation irréductible de niveau zéro de $D^{\times}$de caractère central trivial sur $\varpi^{\mathbb{Z}}$ telle que $\mathrm{JL}(\rho)$ soit une représentation supercuspidale de $G$ par la correspondance de Jacquet-Langlands. Alors, en tant que representations de $G \times W_{K}$, on a

$$
\operatorname{Hom}_{D^{\times}}\left(\rho, H_{c}^{i}\left(\mathcal{M}_{D r, 1} / \varpi^{\mathbb{Z}}, \overline{\mathbb{Q}}_{\ell}\right)\right) \cong \begin{cases}\mathrm{JL}(\rho) \otimes L(\mathrm{JL}(\rho)), & \text { si } i=d-1 \\ 0, & \text { sinon }\end{cases}
$$

Notre preuve repose sur l'étude de la géométrie de $\Sigma_{1}$. Rappelons qu'il existe une application $\tau: \Omega^{d-1} \longrightarrow|\mathcal{B T}|$, où $|\mathcal{B} \mathcal{T}|$ désigne la réalisation géométrique de l'immeuble de Bruhat-Tits semi-simple $\mathcal{B} \mathcal{T}$ associé à $G$. En prenant la composition avec la transition $p: \Sigma_{1} \rightarrow \Omega_{K}^{d-1}$, on obtient un morphisme $\nu: \Sigma_{1} \longrightarrow|\mathcal{B T}|$. Comme il n'existe pas de notion de "base de Drinfeld" à l'instant, on construit sur chaque sommet $s \in \mathcal{B T}$ un modèle entier lisse de $\nu^{-1}(|s|)$ dans (2.3.8), et on obtient en particulier le résultat suivant :

Théorème B. (Théorème (2.5.6)) Si s est un sommet de $\mathcal{B T}$, on a un isomorphisme :

$$
H_{c}^{q}\left(\nu^{-1}\left(|s|^{*}\right), \Lambda\right) \stackrel{\sim}{\longrightarrow} H_{c}^{q}\left(\mathrm{DL}_{\overline{\mathbb{F}}_{q}}^{d-1}, \Lambda\right)
$$

où $|s|^{*}=\cup_{s \in \sigma}|\sigma|$ et $\mathrm{DL}_{\overline{\mathbb{F}}_{q}}^{d-1}$ désigne le revêtement de Deligne-Lusztig Coxeter associé à $\mathrm{GL}_{d}\left(\mathbb{F}_{q}\right)$,

Ce travail est inspiré par celui de Genestier [Gen] sur l'espace de Drinfeld modéré lorsque $K$ est de caractéristique positive, et par celui de Yoshida [Yos10] sur le niveau modéré de la tour de Lubin-Tate. Notre résultat est néanmoins plus précis que celui de Yoshida qui ne décrit que l'action de $G$ et du groupe d'inertie. Notre démonstration est inspirée par Teitelbaum [Tei90] qui étudie la géométrie de $\Sigma_{1}$ lorsque $d=2$. Dans [Str08], Strauch a étudié la correspondance de Jacquet-Langlands dans la tour de Lubin-Tate. 
Nous décrivons brièvement les contenus de différents paragraphes. Dans les paragraphes 2.1 et 2.2 , on rappelle l'espace de Drinfeld $\Omega^{d-1}$ et son revêtement modéré $\Sigma_{1}$. On démontre au paragraphe 2.2, en employant un lemme de Zheng [Zhe08], que l'étude de la cohomologie sans support $H^{q}\left(\nu^{-1}\left(|s|^{*}\right), \Lambda\right)$ se ramène à celle de $H^{q}\left(\nu^{-1}(|s|), \Lambda\right)$. Au paragraphe 2.3, on construit un modèle entier lisse de $\nu^{-1}(|s|)$, défini sur une extension modérément ramifiée du complété de l'extension non ramifiée maximale de $K$, dont la fibre spéciale $\bar{\Sigma}_{s}^{0}$ calcule la cohomologie sans support de $\nu^{-1}(|s|)$. Au cours de la preuve, on utilise la classification, dûe à Raynaud [Ray74], des schémas en groupes de type $(p, \ldots, p)$. Aux paragraphes 2.4 et 2.5, on démontre que $\bar{\Sigma}_{s}^{0} \cong \mathrm{DL}_{\overline{\mathbb{F}}_{q}}^{d-1}$ (et en conséquence le Théorème B.), en calculant leurs classes de $\mu_{q^{d-1}}$-torseur dans $H^{1}\left(\Omega_{\mathbb{F}_{q}}^{d-1}, \mu_{q^{d}-1}\right)$, où $\Omega_{\mathbb{F}_{q}}^{d-1}$ désigne l'espace de Drinfeld sur le corps fini $\mathbb{F}_{q}$. Le théorème A. est alors obtenu dans la section 3. Pour réaliser la correspondance de Langlands locale dans la cohomologie, on a besoin d'un résultat sur les composantes connexes de $\Sigma_{1}$. Ce résultat étant connu dans le cas d'égales caractéristiques [Gen96], peut être obtenu par le travail de Chen [Che13] sur le côté de Lubin-Tate, en vertu d'isomorphisme de Faltings-Fargues, et il nous permet de descendre la fibre spéciale $\bar{\Sigma}_{s}^{0}$, et donc de décrire l'action de Frobenius.

Remerciements : Je remercie profondément mon directeur de thèse Jean-François Dat de me proposer ce sujet et ses constants encouragements. Je tiens à exprimer ma gratitude à Miaofen Chen, Bas Edixhoven, Jared Weinstein et Weizhe Zheng pour leurs enrichissantes discussions. Je remercie Jared Weinstein pour son intérêt sur ce travail et pour m'avoir invité à Boston. Enfin, je remercie le referee anonyme pour ses commentaires.

\section{Sur le revêtement modéré de l'espace symétrique de Drinfeld}

Dans cette section, on rappelle tout d'abord l'espace symétrique $p$-adique de Drinfeld introduit dans [Dri74]. C'est un espace rigide-analytique dont un modèle entier paramètre certains groupes $p$-divisibles [Dri76]. On rappelle le niveau modéré de la tour de Drinfeld. Le lecteur pourra consulter [BC91] pour une démontration détaillée de l'interpretation modulaire lorsque $d=2$. Ensuite, on donne des résultats sur la géométrie du niveau modéré.

\subsection{Rappels sur l'espace symétrique de Drinfeld}

(2.1.1) Soit $d \geqslant 2$ un entier. Fixons un corps $p$-adique $K$, une extension finie de $\mathbb{Q}_{p}$, et notons $\mathcal{O}$ son anneau des entiers et $\varpi$ une uniformisante de $\mathcal{O}$. Le corps résiduel $\mathcal{O} / \varpi \simeq \mathbb{F}_{q}$ est une extension finie de $\mathbb{F}_{p}$, son degré $\left[\mathbb{F}_{q}: \mathbb{F}_{p}\right]$ sera noté $f$. On fixe $K^{c a}$ une clôture algébrique de $K$ et $\widehat{K^{c a}}$ son complété par l'unique norme qui étend celle de $K$. Soient $D$ l'algèbre à division centrale sur $K$ d'invariant $1 / d$ et $\mathcal{O}_{D}$ l'anneau des entiers de $D$. Soient $K_{d}$ une extension non-ramifiée de degré $d$ de $K$ contenue dans $D, \mathcal{O}_{d}$ l'anneau des entiers de $K_{d}$. Il existe un élément $\Pi_{D} \in \mathcal{O}_{D}$ tel que $\mathcal{O}_{D}$ soit engendré sur $\mathcal{O}_{d}$ par $\Pi_{D}$ vérifiant les relations $\Pi_{D}^{d}=\varpi$ et $\Pi_{D} a=\sigma(a) \Pi_{D}, \forall a \in \mathcal{O}_{d}$, où $\sigma \in \operatorname{Gal}\left(K_{d} / K\right)$ le relèvement de Frobenius. On note $\breve{K}$ le complété de l'extension non-ramifiée maximale de $K$ dans la clôture algébrique $K^{c a}$, $\breve{\mathcal{O}}$ son anneau des entiers, on a donc $\breve{K}=\breve{\mathcal{O}}[1 / p]$.

Notons $\mathcal{B} \mathcal{T}$ l'immeuble de Bruhat-Tits semi-simple de $G:=\mathrm{GL}_{d}(K)$, c'est un complexe simplicial dont l'ensemble de sommets s'identifie à l'ensemble des classes d'homothétie de $\mathcal{O}$-réseaux dans l'espace vectoriel $K^{d}$. Un ensemble de sommets $\sigma=\left\{s_{0}, \ldots, s_{k}\right\}$ forme un 
$k$-simplexe s'il existe des représentants $\Lambda_{i}$ de $s_{i} \forall 0 \leqslant i \leqslant k$ tels que $\varpi \Lambda_{k} \subsetneq \Lambda_{0} \subsetneq \cdots \subsetneq \Lambda_{k}$. On notera $\mathcal{B} \mathcal{T}_{k}$ l'ensemble des $k$-simplexes. On désignera $|\mathcal{B T}|$ la réalisation géométrique de $\mathcal{B T}$. Pour $\sigma=\left\{\varpi \Lambda_{k} \subsetneq \Lambda_{0} \subsetneq \cdots \subsetneq \Lambda_{k}\right\}$ un $k$-simplexe de $\mathcal{B} \mathcal{T}$, notons $|\sigma| \subset|\mathcal{B T}|$ sa facette associée : on a pour toute paire de simplexes $\sigma^{\prime} \subset \sigma,\left|\sigma^{\prime}\right| \subset \overline{|\sigma|}$, où $\overline{|\sigma|}$ désigne l'adhérence de $|\sigma|$. On notera aussi $|\sigma|^{*} \subset|\mathcal{B T}|$ la réunion de toutes les facettes $\left|\sigma^{\prime}\right|$ avec $\sigma^{\prime}$ contenant $\sigma$, i.e. $|\sigma|^{*}=\bigcup_{\sigma \subset \sigma^{\prime}}\left|\sigma^{\prime}\right|$. Évidemment, si $\sigma=\left\{s_{0}, \ldots, s_{k}\right\}$ avec $s_{i} \in \mathcal{B} \mathcal{T}_{0}$ des sommets, on a $|\sigma|^{*}=\bigcap_{i}\left|s_{i}\right|^{*}$.

Notons $\Omega_{K}^{d-1}$ l'espace symétrique de Drinfeld de dimension $d-1$, défini dans [Dri74] comme un sous $K$-espace rigide-analytique de l'espace projectif $\mathbb{P}_{K}^{d-1}$. Il s'identifie au complémentaire de l'ensemble des hyperplans $K$-rationnels dans $\mathbb{P}_{K}^{d-1}$. On sait que les points de $|\mathcal{B T}|$ s'identifient aux classes d'homothétie de normes sur le $K$-espace vectoriel $K^{d}$ (cf. [GI63]). Ceci nous fournit une application de réduction $\tau: \Omega_{K}^{d-1} \rightarrow|\mathcal{B T}|$ (voir [DH87]). On désignera par le même symbole $\Omega_{K}^{d-1}$ le $K$-espace analytique $\grave{a}$ la Berkovich correspondant, et il est naturellement muni d'une action continue de $G$ triviale sur le centre ( $c f$. [Ber95]). On obtient alors un espace analytique $\Omega_{K}^{d-1, c a}:=\Omega_{K}^{d-1} \widehat{\otimes}_{K} \widehat{K^{c a}}$ muni d'une action continue du groupe de Weil $W_{K}$ de $K$ en étendant les scalaires à $\widehat{K^{c a}}$.

(2.1.2) Dans un travail non publié, Deligne introduit un modèle semi-stable $\widehat{\Omega}_{\mathcal{O}}^{d-1}$ de $\Omega_{K}^{d-1}$ sur $\operatorname{Spf} \mathcal{O}$, en recollant les modèles locaux (au-dessus des simplexes de $\mathcal{B} \mathcal{T}$ ) que nous rappelons ci-dessous ( $c f$. [Rap90]).

On note alors $\widehat{\Omega}_{\mathcal{O}, \sigma}^{d-1}$ le schéma formel classifiant les classes d'isomorphie de diagrammes commutatifs

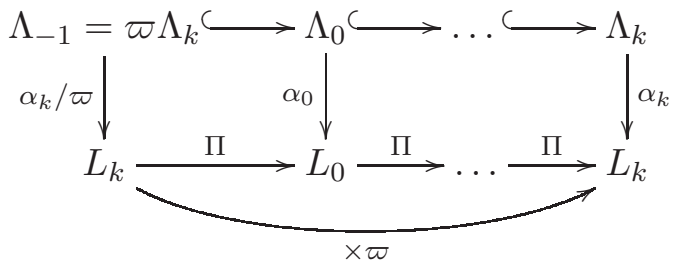

sur un $\mathcal{O}$-schéma $S=\operatorname{Spec}(R)$, où $R \in \operatorname{Nilp}_{\mathcal{O}}$, la catégorie des $\mathcal{O}$-algèbres sur lesquelles $\varpi$ est nilpotent. Les $L_{i}$ sont des fibrés en droites sur $S$, les applications $\alpha_{i}$ sont des homomorphismes de $\mathcal{O}$-modules, les morphismes $\Pi$ sont des homomorphismes de $\mathcal{O}_{S}$-modules, vérifiant la condition : pour tout $x \in \operatorname{Spec}(R)$, on a

$$
\operatorname{Ker}\left\{\alpha_{i}(x): \Lambda_{i} / \varpi \Lambda_{i} \rightarrow L_{i} \otimes_{R} k(x)\right\} \subset \Lambda_{i-1} / \varpi \Lambda_{i},
$$

où $k(x)$ est le corps résiduel de $x$. L'objet universel sur $\widehat{\Omega}_{\mathcal{O}, \sigma}^{d-1}$ sera noté :

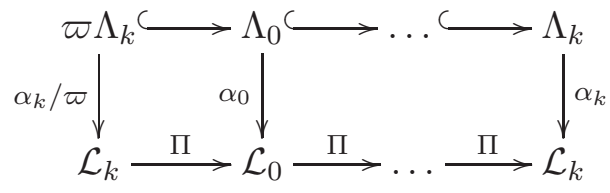

FAIT.- (cf. [Gen96, (III.1)]) On note $\widetilde{\mathbb{P}}_{\sigma}$ le $\mathcal{O}$-schéma obtenu à partir de $\mathbb{P}\left(\Lambda_{k}\right)=\operatorname{Proj}\left(\operatorname{Sym}\left(\Lambda_{k}\right)\right)$ en l'éclatant successivement le long du sous-schéma fermé $\mathbb{P}\left(\Lambda_{k} / \Lambda_{k-1}\right)$ de sa fibre spéciale $\widetilde{\mathbb{P}}_{\sigma} \otimes \overline{\mathbb{F}}_{q}=\mathbb{P}\left(\Lambda_{k} / \varpi \Lambda_{k}\right)$, puis en éclatant le transformé strict de $\mathbb{P}\left(\Lambda_{k} / \Lambda_{k-2}\right)$, puis le transformé strict de $\mathbb{P}\left(\Lambda_{k} / \Lambda_{k-3}\right)$ et ainsi de suite. Comme expliqué dans [Gen96, (III.1)], $\widetilde{\mathbb{P}}_{\sigma}$ représente un foncteur $H_{\sigma}$ sur Nilp $_{\mathcal{O}}$ qui associe à $R \in$ Nilp $_{\mathcal{O}}$ l'ensemble des classes d'isomorphie de diagrammes commutatifs 


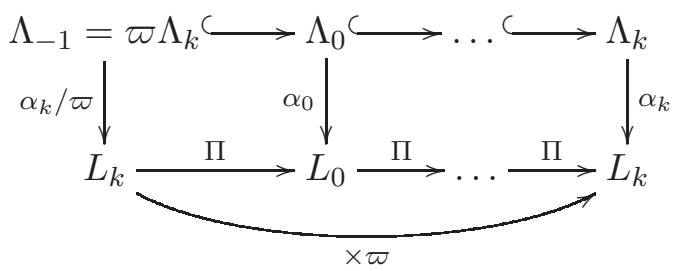

où les $L_{i}$ sont des $R$-modules inversibles, les applications $\alpha_{i}$ sont des homomorphismes de $\mathcal{O}$ modules, les morphismes $\Pi$ sont des homomorphismes de $R$-modules, tels que pour tout $i$ le morphisme $B$-linéaire

$$
\alpha_{i} \otimes \operatorname{Id}_{R}: \Lambda_{i} \otimes_{\mathcal{O}} R \longrightarrow L_{i}
$$

soit surjectif. On a alors une immersion de $\widehat{\Omega}_{\mathcal{O}, \sigma}^{d-1}$ dans $\widetilde{\mathbb{P}}_{\sigma}$. On désigne $\Omega_{\mathcal{O}, \sigma}^{0}$ le complémentaire dans la fibre spéciale $\widetilde{\mathbb{P}}_{\sigma} \otimes \overline{\mathbb{F}}_{q}$ de $\widetilde{\mathbb{P}}_{\sigma}$ des fermés

$$
\left\{\alpha_{j}(m)=0\right\} \quad\left(j \in \mathbb{Z} / k \mathbb{Z}, m \in \Lambda_{j} / \varpi \Lambda_{j}-\Lambda_{j-1} / \varpi \Lambda_{j}\right) .
$$

Alors $\widehat{\Omega}_{\mathcal{O}, \sigma}^{d-1}$ s'identifie au complété de $\widetilde{\mathbb{P}}_{\sigma}$ le long de $\Omega_{\mathcal{O}, \sigma}^{0}$. Lorsque $\sigma^{\prime}$ est un sous-simplexe de $\sigma$, on a une immersion naturelle ouverte $\widehat{\Omega}_{\mathcal{O}, \sigma^{\prime}}^{d-1} \hookrightarrow \widehat{\Omega}_{\mathcal{O}, \sigma}^{d-1}$. Le schéma formel $\widehat{\Omega}_{\mathcal{O}}^{d-1}$ est par définition la limite inductive $\underset{\sigma \in \mathcal{B} \mathcal{T}}{\lim } \widehat{\Omega}_{\mathcal{O}, \sigma}^{d-1}$. De plus, la fibre générique de $\widehat{\Omega}_{\mathcal{O}}^{d-1}$ (au sens de Raynaud-Berkovich) s'identifie à $\Omega_{K}^{d-1}$. Si $g \in G$ envoyant un simplexe $\sigma$ sur $g \sigma$, on définit un isomorphisme $g: \widehat{\Omega}_{\mathcal{O}, \sigma}^{d-1} \rightarrow \widehat{\Omega}_{\mathcal{O}, g \sigma}^{d-1}$ en associant à une donnée $\left(\alpha_{j}: \Lambda_{j} \rightarrow L_{j}, \Pi\right)$ la donnée $\left(g \Lambda_{j} \stackrel{g^{-1}}{\longrightarrow} \Lambda_{j} \stackrel{\alpha_{j}}{\longrightarrow} L_{j}, \Pi\right)$. Ceci définit une action de $G$ sur le système inductif $\left(\widehat{\Omega}_{\mathcal{O}, \sigma}^{d-1}\right)_{\sigma}$, et donc sur $\Omega_{K}^{d-1}$. Sous cette action, l'application de réduction $\tau: \Omega_{K}^{d-1} \rightarrow|\mathcal{B} \mathcal{T}|$ est $G$-équivariante.

(2.1.3) Définition.- Soit $\sigma=\left\{\varpi \Lambda_{k} \subsetneq \Lambda_{0} \subsetneq \cdots \subsetneq \Lambda_{k}\right\}$ un $k$-simplexe, le type de $\sigma$ est la suite des entiers $\left(e_{0}, \ldots, e_{k}\right)$ telle que $e_{0}=\operatorname{dim}_{\mathbb{F}_{q}} \Lambda_{0} / \varpi \Lambda_{k}$ et $e_{i}=\operatorname{dim}_{\mathbb{F}_{q}} \Lambda_{i} / \Lambda_{i-1}$ pour $1 \leqslant i \leqslant k$. Évidemment, on a $\sum e_{i}=d$.

(2.1.4) Exemple.- Nous aurons besoin des descriptions explicites suivantes.

Commençons par le cas où le simplexe $\sigma=\Phi$ est maximal, i.e. $\Phi$ est associé à une chaîne des réseaux $\varpi \Phi_{d-1} \subsetneq \Phi_{0} \subsetneq \cdots \subsetneq \Phi_{d-1}$. On peut supposer que $\Phi_{d-1}=\mathcal{O}^{d}$, et que sous la base canonique $\left\{e_{0}, \ldots, e_{d-1}\right\}$ de $\mathcal{O}^{d}$,

$$
\Phi_{i}=\left\langle e_{0}, \ldots, e_{i}, \varpi e_{i+1}, \ldots, \varpi e_{d-1}\right\rangle, \forall 0 \leqslant i \leqslant d-1 .
$$

La condition imposée sur $\alpha_{i}$ implique que $\alpha_{i}\left(e_{i}\right)$ engendre $L_{i}$. Identifions $L_{i}$ avec $R$ en posant $\alpha_{i}\left(e_{i}\right)=1$. Le morphisme $R$-linéaire $\Pi: L_{i} \rightarrow L_{i+1}$ est alors donné par multiplication par $c_{i}$, où

$$
c_{i}=\frac{\alpha_{d-1}\left(e_{i}\right)}{\alpha_{d-1}\left(e_{i+1}\right)}, \quad 0 \leqslant i \leqslant d-2 \text { et } c_{d-1}=\frac{\varpi \alpha_{d-1}\left(e_{d-1}\right)}{\alpha_{d-1}\left(e_{0}\right)} .
$$

Ceci nous permet d'identifier le schéma formel $\widehat{\Omega}_{\mathcal{O}, \Phi}^{d-1}$ au spectre formel du complété $\varpi$-adique de l'anneau

$$
\mathcal{O}\left[c_{0}, \ldots, c_{d-1}, P_{\Phi}^{-1}\right] /\left(\prod c_{i}-\varpi\right),
$$

où $P_{\Phi}=\prod P_{\mathfrak{a}, i}, i \in \mathbb{Z} / d \mathbb{Z}, \mathfrak{a}=\left(a_{0}, \ldots, a_{d-2}\right)$ parcourt une classe de représentants de $(\mathcal{O} / \varpi \mathcal{O})^{d-1}$ dans $\mathcal{O}^{d-1}$, et $P_{\mathfrak{a}, i}=1+a_{0} c_{i-1}+a_{1} c_{i-1} c_{i-2}+\cdots+a_{d-2} c_{i-1} \cdots c_{1+i-d}$. 
L'objet universel sur $\widehat{\Omega}_{\mathcal{O}, \Phi}^{d-1}$ est la donnée d'une suite de $\mathcal{O}_{\widehat{\Omega}_{\mathcal{O}, \Phi}^{d-1}}$-modules

$$
\mathcal{L}_{d-1} \stackrel{\Pi}{\longrightarrow} \mathcal{L}_{0} \stackrel{\Pi}{\longrightarrow} \cdots \stackrel{\Pi}{\longrightarrow} \mathcal{L}_{d-1}
$$

avec $\mathcal{L}_{i}$ libre de base 1 et $\Pi: \mathcal{L}_{i} \rightarrow \mathcal{L}_{i+1}$ est la multiplication par $c_{i}$.

Ensuite on considère le cas où $\sigma=[\Lambda]$ est un sommet. Il suffit de traiter le cas où $\Lambda=\mathcal{O}^{d}$. Le schéma formel $\widehat{\Omega}_{\mathcal{O},[\Lambda]}^{d-1}$ classifie les diagrammes commutatifs

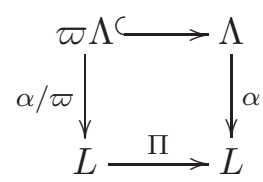

tels que l'application

$$
\alpha(x): \Lambda / \varpi \Lambda \longrightarrow L \otimes_{R} k(x)
$$

soit injective pour tout $x \in \operatorname{Spec}(R)$. Ceci implique que $\alpha(u)$ est un générateur de $L, \forall u \in$ $\Lambda \backslash \varpi \Lambda$. Le couple $(L, \alpha)$ est alors déterminé à isomorphisme près par

$$
\left(x_{0}=\frac{\alpha\left(e_{0}\right)}{\alpha\left(e_{d-1}\right)}, \ldots, x_{d-2}=\frac{\alpha\left(e_{d-2}\right)}{\alpha\left(e_{d-1}\right)}\right) \in R^{d-1} .
$$

Nous pouvons donc identifier le schéma formel $\widehat{\Omega}_{\mathcal{O},[\Lambda]}^{d-1}$ au spectre formel du complété $\varpi$-adique de l'anneau

$$
\mathcal{O}\left[x_{0}, \ldots, x_{d-2}, P_{\Lambda}^{-1}\right]
$$

où $P_{\Lambda}=\prod\left(a_{0} x_{0}+a_{1} x_{1}+\cdots+a_{d-2} x_{d-2}+a_{d-1}\right),\left(a_{0}, \ldots, a_{d-1}\right)$ parcourt une classe de représentants de $(\mathcal{O} / \varpi \mathcal{O})^{d} \backslash\{0\}$ dans $\mathcal{O}^{d}$. L'objet universel sur $\widehat{\Omega}_{\mathcal{O},[\Lambda]}^{d-1}$ est la donnée d'un $\mathcal{O}_{\widehat{\Omega}_{\mathcal{O},[\Lambda]}^{d-1}}$ module $\mathcal{L}$ libre de base 1 , et $\Pi: \mathcal{L} \rightarrow \mathcal{L}$ est la multiplication par $\varpi$.

REMARqUE.- L'immersion canonique $\widehat{\Omega}_{\mathcal{O},[\Lambda]}^{d-1} \hookrightarrow \widehat{\Omega}_{\mathcal{O}, \Phi}^{d-1}\left(\right.$ ou $\Omega_{K,[\Lambda]}^{d-1} \hookrightarrow \Omega_{K, \Phi}^{d-1} \hookrightarrow \mathbb{P}_{K}^{d-1}$ ) induit une identification $c_{i}=x_{i} / x_{i+1}$ pour $0 \leqslant i \leqslant d-3, c_{d-2}=x_{d-2}, c_{d-1}=\varpi / x_{0}$.

Enfin, soit $\sigma=\left\{\varpi \Lambda_{d-1} \subsetneq \Lambda_{0} \subsetneq \Lambda_{d-1}=\mathcal{O}^{d}\right\}$ le sous-simplexe de $\Phi$ de type $(1, d-1)$, i.e. $\Lambda_{0}$ est engendré par $e_{0}, \varpi e_{1}, \ldots, \varpi e_{d-1}$. L'objet universel correspondant est décrit par le diagramme commutatif suivant

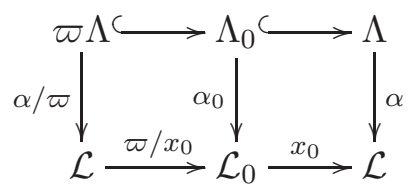

Le schéma formel $\widehat{\Omega}_{\mathcal{O}, \sigma}^{d-1}$ s'identifie au spectre formel du complété $\varpi$-adique de l'anneau

$$
\mathcal{O}\left[x_{0}, \ldots, x_{d-2}, c_{d-1}, P_{\sigma}^{-1}\right] /\left(x_{0} c_{d-1}-\varpi\right)
$$

où $P_{\sigma}=\prod\left(1+a_{0} x_{0}+\cdots+a_{d-2} x_{d-2}\right)\left(1+a_{0} x_{1} c_{d-1}+\cdots+a_{d-3} x_{d-2} c_{d-1}+a_{d-2} c_{d-1}\right),\left(a_{0}, \ldots, a_{d-2}\right)$ parcourt une classe de représentants de $(\mathcal{O} / \varpi \mathcal{O})^{d-1}$ dans $\mathcal{O}^{d-1}$. 
(2.1.5) Les composantes irréductibles de la fibre spéciale géométrique $\bar{\Omega}:=\widehat{\Omega}_{\mathcal{O}}^{d-1} \otimes_{\mathcal{O}} \overline{\mathbb{F}}_{q}$ de $\widehat{\Omega}_{\mathcal{O}}^{d-1}$ sont paramétrées par les sommets de $\mathcal{B T}$. Plus précisément, soit $s=\left[\Lambda_{s}\right]$ représenté par un réseau $\Lambda_{s}$, considérons tous les simplexes maximaux contenant $s$. Pour un tel simplexe $\sigma$, il est représenté par une suite de réseaux $\left\{\varpi \Lambda_{s} \subsetneq \Lambda_{\sigma, 0} \subsetneq \cdots \subsetneq \Lambda_{\sigma, d-2} \subsetneq \Lambda_{s}\right\}$. Notons $\bar{\Omega}_{s}$ la variété projective obtenue à partir de $\mathbb{P}\left(\Lambda_{s} / \varpi \Lambda_{s}\right)$ en l'éclatant successivement le long du sous-schéma fermé $\mathbb{P}\left(\Lambda_{s} / \Lambda_{\sigma, d-2}\right)$ pour tout simplexe maximal $\sigma$ contenant $s$, puis en éclatant le transformé strict de $\mathbb{P}\left(\Lambda_{s} / \Lambda_{\sigma, d-3}\right)$ pour tous ces $\sigma$, puis le transformé strict de $\mathbb{P}\left(\Lambda_{s} / \Lambda_{\sigma, d-4}\right)$ pour tous ces $\sigma$ et ainsi de suite, $c f$. [Ito05, $\S 4]$ ou [Wan13, (4.1.2)]. On sait alors que le $\overline{\mathbb{F}}_{q^{-}}$ schéma $\bar{\Omega}$ est localement de type fini, et $\bar{\Omega}=\bigcup_{s \in \mathcal{B} \mathcal{T}_{0}} \bar{\Omega}_{s}$. Chaque $\bar{\Omega}_{s}$ est une variété projective munie d'une action de $G_{s}:=\operatorname{Stab}_{G}(s)$. Soit $\sigma=\left\{s_{0}, \ldots, s_{k}\right\}$ un simplexe quelconque, notons $\bar{\Omega}_{\sigma}$ la variété projective $\bar{\Omega}_{s_{0}} \cap \cdots \cap \bar{\Omega}_{s_{k}}$ munie d'une action de $\widehat{G_{\sigma}}:=\operatorname{Stab}_{G}(\sigma)$. On désigne $G_{\sigma}$ le fixateur de $\sigma$, et $G_{\sigma}^{+}$le pro-p-radical de $G_{\sigma}$ (voir [SS93]). Notons $\bar{\Omega}_{\sigma}^{0}:=\bar{\Omega}_{\sigma} \backslash \bigcup_{s^{\prime} \notin \sigma} \bar{\Omega}_{s^{\prime}}$, et $j_{\sigma}: \bar{\Omega}_{\sigma}^{0} \hookrightarrow \bar{\Omega}_{\sigma}$ l'inclusion naturelle. En particulier, $\bar{\Omega}_{s}^{0}$ est la fibre spéciale géométrique de $\widehat{\Omega}_{\mathcal{O}, s}^{d-1}$ (le modèle de Deligne au-dessus de $s$ ).

Rappelons que Berkovich a défini dans ce cas un morphisme de spécialisation

$$
\mathrm{sp}: \Omega_{K}^{d-1, c a} \longrightarrow \bar{\Omega}
$$

(qui est appelé le morphisme de réduction dans [Ber96, §1]). L'image réciproque sous ce morphisme de l'inclusion naturelle $j_{s}: \bar{\Omega}_{s}^{0} \hookrightarrow \bar{\Omega}_{s}$ s'identifie à :

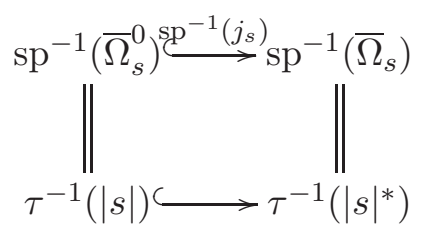

(2.1.6) Lemme.- Soit s un sommet. Quitte à choisir une base d'un réseau qui représente $s$, on a un isomorphisme $G_{s} / G_{s}^{+} \stackrel{\sim}{\longrightarrow} \mathrm{GL}_{d}\left(\mathbb{F}_{q}\right)$. Via cet isomorphisme, $\bar{\Omega}_{s}^{0}$ munie de l'action de $G_{s} / G_{s}^{+}\left(G_{s}^{+}\right.$agit trivialement sur $\left.\bar{\Omega}_{s}^{0}\right)$ est isomorphe à $\Omega_{\overline{\mathbb{F}}_{q}^{d}}^{d-1}$ muni de l'action de $\mathrm{GL}_{d}\left(\mathbb{F}_{q}\right)$, où $\Omega_{\overline{\mathbb{F}}_{q}}^{d-1}$ est le complémentaire de tous les hyperplans $\mathbb{F}_{q^{-}}$-rationnels dans $\mathbb{P}_{\overline{\mathbb{F}}_{q}}^{\text {d-1 appelé l'espace }}$ de Drinfeld sur le corps fini $\mathbb{F}_{q}$ (cf. (2.5.1)).

Preuve : On peut supposer que $s=[\Lambda]=\left[\mathcal{O}^{d}\right]$ le réseau standard. D'après l'exemple (2.1.4), $\bar{\Omega}_{s}^{0}=\operatorname{Spec} \overline{\mathbb{F}}_{q}\left[x_{0}, \ldots, x_{d-2}, \bar{P}_{\Lambda}^{-1}\right]$ qui s'identifie donc à $\Omega_{\overline{\mathbb{F}}_{q}}^{d-1}$. Les éléments de $G_{s}^{+}=1+\varpi M_{d}(\mathcal{O})$ agissent bien trivialement, et les actions sont compatibles.

\subsection{Le revêtement modéré $\Sigma^{c a}$}

(2.2.1) Rappelons tout d'abord deux descriptions modulaires de notre schéma formel $\widehat{\Omega}_{\mathcal{O}}^{d-1}$ introduites par Drinfeld dans [Dri76] (voir aussi [BC91]). Si $R$ est une $\mathcal{O}$-algèbre, nous noterons $R[\Pi]$ le quotient de l'algèbre de polynômes $R[X]$ par l'idéal engendré par $X^{d}-\varpi$. C'est donc un $R$-module libre de rang $d$, engendré par 1 et un élément $\Pi$ (l'image de $X$ ) qui vérifie $\Pi^{d}=\varpi$. L'algèbre $R[\Pi]$ est munie d'une graduation à valeurs dans $\mathbb{Z} / d \mathbb{Z}$ telle que les éléments de $R$ soient de degré 0 , et $\Pi$ soit de degré 1 .

On considère le foncteur $F^{D r}$ qui associe à une algèbre $R \in$ Nilp $_{\mathcal{O}}$ l'ensemble des classes d'isomorphie de $(\psi, \eta, T, u, r)$, où 
- $\psi$ est un $\mathbb{F}_{q}$-homomorphisme de $\overline{\mathbb{F}}_{q}$ vers $R / \varpi R$.

- $\eta$ est un faisceau en $\mathcal{O}[\Pi]$-modules plats, $\mathbb{Z} / d \mathbb{Z}$-gradué, constructible, sur $S:=\operatorname{Spec}(R)$ muni de la topologie de Zariski.

- $T$ est un faisceau en $\mathcal{O}_{S}[\Pi]$-modules, $\mathbb{Z} / d \mathbb{Z}$-gradué, tel que les composantes homogènes soient des faisceaux inversibles sur $S$.

- $u$ est un homomorphisme $\mathcal{O}[\Pi]$-linéaire de degré 0 de $\eta$ vers $T$, tel que $u \otimes_{\mathcal{O}} \mathcal{O}_{S}: \eta \otimes_{\mathcal{O}}$ $\mathcal{O}_{S} \rightarrow T$ soit surjectif.

- $r$ est un isomorphisme $K$-linéaire du faisceau constant $\underline{K}^{d}$ vers le faisceau $\eta_{0} \otimes_{\mathcal{O}} K$.

satisfaisant les conditions suivantes :

- Soit $S_{i} \subset S$ le lieu d'annulation du morphisme $\Pi: T_{i} \rightarrow T_{i+1}$, alors la restriction $\left.\eta_{i}\right|_{S_{i}}$ est un faisceau constant de fibre isomorphe à $\mathcal{O}^{d}$.

- Pour tout point $s \in S$ l'application $\eta_{s} / \Pi \eta_{s} \rightarrow\left(T_{s} / \Pi T_{s}\right) \otimes k(s)$ est injective, où $k(s)$ est le corps résiduel de $s$.

- $\left.\bigwedge^{d}\left(\eta_{i}\right)\right|_{S_{i}}=\left.\varpi^{-i}\left(\bigwedge^{d}\left(\Pi^{i} r \underline{\mathcal{O}}^{d}\right)\right)\right|_{S_{i}}(\forall i \in \mathbb{Z} / d \mathbb{Z})$.

Drinfeld démontre que ce foncteur $F^{D r}$ est pro-représentable par le $\mathcal{O}$-schéma formel $\widehat{\Omega}_{\breve{\mathcal{O}}}^{d-1}:=\widehat{\Omega}_{\mathcal{O}}^{d-1} \widehat{\otimes}_{\mathcal{O}} \breve{\mathcal{O}}$. Dans la suite, on notera $(\boldsymbol{\psi}, \boldsymbol{\eta}, \mathbf{T}, \mathbf{u}, \mathbf{r})$ l'objet universel sur $\widehat{\Omega}_{\breve{\mathcal{O}}}^{d-1}$. Les composantes homogènes universelles $\mathbf{T}_{i}$ sont des fibrés en droites sur $\widehat{\Omega}_{\breve{\mathcal{O}}}^{d-1}$. Par la construction de l'isomorphisme entre $\widehat{\Omega}_{\breve{\mathcal{O}}}^{d-1}$ et $F^{D r}$ ( $c f$. [BC91]), on sait que la restriction de $\mathbf{T}_{i}$ à chaque $\widehat{\Omega}_{\breve{\mathcal{O}}, \sigma}^{d-1}:=\widehat{\Omega}_{\mathcal{O}, \sigma}^{d-1} \widehat{\otimes}_{\mathcal{O}} \breve{\mathcal{O}}$ est en fait libre. Soit $\sigma=\Phi$ le simplexe maximal standard, et identifions $\left.\mathbf{T}_{i}\right|_{\widehat{\Omega}_{\breve{\mathcal{O}}, \Phi}^{d-1}}$ avec $\mathcal{O}_{\widehat{\Omega}}^{\widehat{\Omega}_{\mathscr{\mathcal { O }}, \Phi}^{d-1}}$. Via cette identification, l'application $\Pi: \mathbf{T}_{i} \rightarrow \mathbf{T}_{i+1}$ est induite par multiplication par $c_{i}$ (cf. l'exemple (2.1.4)).

Le foncteur $G^{D r}$ de Drinfeld est un problème de modules des $\mathcal{O}_{D}$-modules formels munis d'une certaine rigidification. Rappelons ci-dessous leurs définitions. Si $R$ est une $\mathcal{O}$-algèbre, un $\mathcal{O}$-module formel $X$ est un groupe formel sur $R$ muni d'une action de $\mathcal{O}$ relevant l'action naturelle sur l'espace tangent $\operatorname{Lie}(X)$. Un $\mathcal{O}_{D}$-module formel sur $R$ est un $\mathcal{O}$-module formel muni d'une action de $\mathcal{O}_{D}$ prolongeant l'action de $\mathcal{O}$. Un $\mathcal{O}_{D}$-module formel $X$ est dit spécial si l'action de $\mathcal{O}_{d}$ fait de $\operatorname{Lie}(X)$ un $\mathcal{O}_{d} \otimes_{\mathcal{O}} R$-module localement libre de rang 1.

La définition du foncteur $G^{D r}$ repose sur l'existence d'un $\mathcal{O}_{D}$-module formel spécial $\mathbb{H}$ de dimension $d$ et $(\mathcal{O}-)$ hauteur $d^{2}$ sur $\overline{\mathbb{F}}_{q}$, qui est unique à isogénie près ( $c f$. [Dri76] voir aussi [BC91, II Prop. 5.2]). On considère le foncteur $G^{D r} \operatorname{sur}^{\text {Nilp }} \mathrm{P}_{\mathcal{O}}$ qui associe à $R \in \mathrm{Nilp}_{\mathcal{O}}$ l'ensemble des classes d'isomorphie de triple $(\psi, X, \rho)$, où

- $\psi$ est un $\mathbb{F}_{q}$-homomorphisme de $\overline{\mathbb{F}}_{q}$ vers $R / \varpi R$.

- $X$ est un $\mathcal{O}_{D}$-module formel spécial de hauteur $d^{2}$ sur $R$.

- $\rho$ est une quasi-isogénie de hauteur zéro de $\psi^{*} \mathbb{H}:=\mathbb{H} \otimes_{\overline{\mathbb{F}}_{q}, \psi} R / \varpi R$ vers $X_{R / \varpi R}$.

Un théorème difficile de Drinfeld nous dit qu'il existe un isomorphisme entre $G^{D r}$ et $F^{D r}$. C'est-à-dire $G^{D r}$ est pro-représentable par le $\mathcal{O}$-schéma formel $\widehat{\Omega}_{\breve{\mathcal{O}}}^{d-1}$.

(2.2.2) On désigne $\mathfrak{X}$ le $\mathcal{O}_{D}$-module formel spécial universel de dimension $d$ et hauteur $d^{2}$ sur $\widehat{\Omega}_{\breve{\mathcal{O}}}^{d-1}$. Le morphisme $\Pi_{D}: \mathfrak{X} \rightarrow \mathfrak{X}$ est une isogénie, son noyau $\mathfrak{X}\left[\Pi_{D}\right]$ est un schéma formel en groupes fini plat de rang $q^{d}$ sur $\widehat{\Omega}_{\breve{\mathcal{O}}}^{d-1}$. On note $\Sigma:=\underline{\operatorname{Isom}}_{\mathcal{O}_{D}}\left(\mathcal{O}_{D} / \Pi_{D} \mathcal{O}_{D}, \mathfrak{X}\left[\Pi_{D}\right]^{\text {rig }}\right)$ ou $\underline{\operatorname{Isom}}_{\mathcal{O}_{D}}\left(\mathcal{O}_{D} / \Pi_{D} \mathcal{O}_{D}, \mathfrak{X}\left[\Pi_{D}\right]^{a n}\right)$ selon besoin. Par construction, $\Sigma$ est un revêtement fini étale sur $\Omega_{\breve{K}}^{d-1}:=\Omega_{K}^{d-1} \widehat{\otimes}_{K} \breve{K}$ de groupe de Galois $\left(\mathcal{O}_{D} / \Pi_{D} \mathcal{O}_{D}\right)^{\times} \simeq \mathbb{F}_{q^{d}}^{\times}$. On note $\Sigma^{c a}:=\Sigma \widehat{\otimes}_{K} \widehat{K^{c a}} \stackrel{p}{\longrightarrow}$ $\Omega_{K}^{d-1, c a}$ la projection naturelle induite par $\mathfrak{X}\left[\Pi_{D}\right] \rightarrow \widehat{\Omega}_{\breve{\mathcal{O}}}^{d-1}$ après une extension de scalaires. On sait que le groupe des quasi-isogénies de hauteur zéro du $\mathcal{O}_{D}$-module formel $\mathbb{H}$ vers lui-même 
s'identifie à

$$
G^{\circ}:=\operatorname{Ker}\left(\operatorname{val}_{K} \circ \operatorname{det}: \mathrm{GL}_{d}(K) \rightarrow K^{\times}\right)
$$

où $\operatorname{val}_{K}$ est la valuation normalisée sur $K$ de sorte que $\operatorname{val}_{K}(\varpi)=1$. Ceci fournit une action de $G^{\circ}$ sur tous les niveaux de la tour de Drinfeld. Par conséquent le morphisme de transition $p: \Sigma^{c a} \rightarrow \Omega_{K}^{d-1, c a}$ est $G^{\circ}$-équivariant. Dans cet article, on s'intéresse à la cohomologie étale à support compact de $\Sigma^{c a}$ au sens de Berkovich.

Par la construction précédente, on a un diagramme commutatif dont toutes les flèches sont $G^{\circ}$-équivariantes :

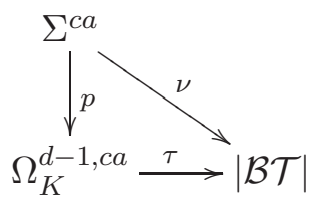

où $\nu$ est la composée $\tau \circ p$. Donc $\Sigma^{c a}$ admet un recouvrement par les ouverts admissibles $\nu^{-1}\left(|s|^{*}\right)$ où $s$ parcourent les sommets de $\mathcal{B} \mathcal{T}$. Soit $s$ un sommet quelconque de $\mathcal{B} \mathcal{T}$, l'immersion ouverte $\nu^{-1}(|s|) \hookrightarrow \nu^{-1}\left(|s|^{*}\right)$ induit un morphisme de restriction :

$$
R \Gamma\left(\nu^{-1}\left(|s|^{*}\right), \Lambda\right) \stackrel{\text { res }}{\longrightarrow} R \Gamma\left(\nu^{-1}(|s|), \Lambda\right)
$$

où $\Lambda=\mathbb{Z} / n$ avec $n$ un entier premier à $p$.

(2.2.3) THÉORÈME.- Le morphisme de restriction ci-dessus est en fait un isomorphisme.

Preuve: La démonstration repose sur un résultat des cycles évanescents d'un faisceau modéré sur une variété de réduction semi-stable établi par Zheng $c f$. [Zhe08, Lemme 5.6]. Supposons tout d'abord que $\Gamma$ soit un sous-groupe discret cocompact et sans torsion de $\mathrm{PGL}_{d}(K)$. On sait alors que $\Gamma$ agit librement sur $\widehat{\Omega}_{\breve{\mathcal{O}}}^{d-1}$ de sorte que $\widehat{\Omega}_{\breve{\mathcal{O}}}^{d-1} / \Gamma$ soit propre sur $\operatorname{Spf} \breve{\mathcal{O}}$. On peut munir $\Omega_{\breve{K}}^{d-1} / \Gamma$ d'une structure de $\breve{K}$-espace analytique telle que le quotient $\Omega_{\breve{K}}^{d-1} \rightarrow \Omega_{\breve{K}}^{d-1} / \Gamma$ soit un revêtement analytique galoisien. D'après Kurihara [Kur80] et Mustafin [Mus78], $\Omega_{\breve{K}}^{d-1} / \Gamma$ est algébrisable, i.e. il existe un schéma propre $X_{\Gamma}$ sur $\breve{\mathcal{O}}$ de réduction semi-stable tel que $\widehat{\Omega}_{\breve{\mathcal{O}}}^{d-1} / \Gamma$ soit le complété de $X_{\Gamma}$ le long de sa fibre spéciale $X_{\Gamma 0}$. La stratification de $X_{\Gamma 0}$ coïncide avec le complexe simplicial $\mathcal{B} \mathcal{T} / \Gamma$ cf. [Kur80, Thm. 2.2.6]. Quitte à rapetisser $\Gamma$, on peut supposer que la projection $\pi: \widehat{\Omega}_{\breve{\mathcal{O}}}^{d-1} \rightarrow \widehat{\Omega}_{\breve{\mathcal{O}}}^{d-1} / \Gamma$ induit un isomorphisme entre $\bar{\Omega}_{s}$ et $\pi\left(\bar{\Omega}_{s}\right)$. Notons $Z$ (resp. $Z^{0}$ ) le sous-schéma localement fermé de $X_{\Gamma}$ qui correspond à $\pi\left(\bar{\Omega}_{s}\right)$ (resp. $\pi\left(\bar{\Omega}_{s}^{0}\right)$ ) par l'algébrisation. D'après GAGA analytique, le revêtement étale modérément ramifié $\Sigma_{\breve{K}} / \Gamma$ de $\Omega_{\breve{K}}^{d-1} / \Gamma$ correspond à un revêtement modéré $f: Y \rightarrow X_{\Gamma, \eta}$ de $X_{\Gamma, \eta}$, où $X_{\Gamma, \eta}$ est la fibre générique de $X_{\Gamma}$.

D'après [Ber96, Corollary 3.5], on a des isomorphismes canoniques :

$$
\begin{aligned}
& R \Gamma\left(\nu^{-1}\left(|s|^{*}\right), \Lambda\right)=R \Gamma\left(\tau^{-1}\left(|s|^{*}\right),\left.p_{*} \Lambda\right|_{\tau^{-1}\left(|s|^{*}\right)}\right) \stackrel{\sim}{\sim} R \Gamma\left(\bar{\Omega}_{s},\left.R \Psi_{\eta}\left(p_{*} \Lambda\right)\right|_{\bar{\Omega}_{s}}\right) \\
& R \Gamma\left(\nu^{-1}(|s|), \Lambda\right)=R \Gamma\left(\tau^{-1}(|s|),\left.p_{*} \Lambda\right|_{\tau^{-1}(|s|)}\right) \stackrel{\sim}{\longrightarrow} R \Gamma\left(\bar{\Omega}_{s}^{0},\left.R \Psi_{\eta}\left(p_{*} \Lambda\right)\right|_{\bar{\Omega}_{s}^{0}}\right)
\end{aligned}
$$

qui nous donnent un diagramme commutatif

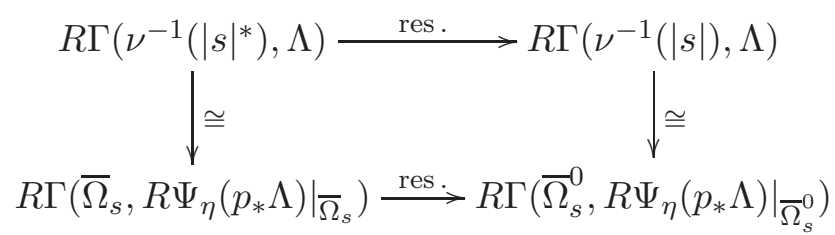


où $R \Psi_{\eta}$ désigne le foncteur des cycles évanescents formels défini par Berkovich dans loc. cit. . Le théorème principal de Berkovich nous dit qu'il existe des isomorphismes canoniques

$$
\left.\left.R \Psi_{\eta}\left(p_{*} \Lambda\right)\right|_{\Omega_{s}} \cong R \Psi\left(X_{\Gamma}, f_{*} \Lambda\right)\right|_{Z}
$$

et

$$
\left.\left.R \Psi_{\eta}\left(p_{*} \Lambda\right)\right|_{\bar{\Omega}_{s}^{0}} \cong R \Psi\left(X_{\Gamma}, f_{*} \Lambda\right)\right|_{Z^{0}},
$$

où $R \Psi$ est le foncteur des cycles évanescents algébrique. En vertu du [Zhe08, Lemme 5.6], nous avons

$$
\left.R \Psi\left(X_{\Gamma}, f_{*} \Lambda\right)\right|_{Z}=\left.R j_{Z *} R \Psi\left(X_{\Gamma}, f_{*} \Lambda\right)\right|_{Z^{0}},
$$

où $j_{Z}$ désigne l'immersion naturelle $Z^{0} \hookrightarrow Z$. On en déduit l'égalité suivante

$$
\left.R \Psi_{\eta}\left(p_{*} \Lambda\right)\right|_{\bar{\Omega}_{s}}=\left.R j_{s, *} R \Psi_{\eta}\left(p_{*} \Lambda\right)\right|_{\bar{\Omega}_{s}^{0}}
$$

et donc un isomorphisme

$$
R \Gamma\left(\nu^{-1}\left(|s|^{*}\right), \Lambda\right) \stackrel{\sim}{\longrightarrow} R \Gamma\left(\nu^{-1}(|s|), \Lambda\right)
$$

donné par le morphisme de restriction.

\subsection{Le prolongement de $\Sigma^{c a}$ au-dessus d'un sommet}

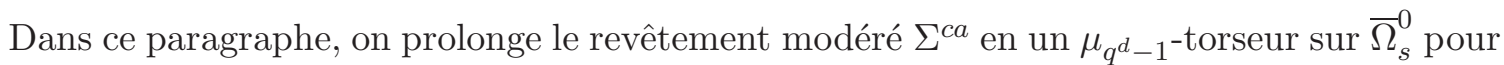
chaque sommet $s$ de $\mathcal{B} \mathcal{T}$. Les calculs que nous effectuons ici généralisent ceux de Teitelbaum [Tei90] pour $d=2$.

(2.3.1) L'espace tangent $\operatorname{Lie}(\mathfrak{X})$ du $\mathcal{O}_{D}$-module formel spécial universel $\mathfrak{X}$ (voir $(2.2 .2)$ ) admet une graduation par $\mathbb{Z} / d \mathbb{Z}$ sous l'action de $\mathcal{O}_{d} \subset \mathcal{O}_{D}$ en posant

$$
\operatorname{Lie}(\mathfrak{X})_{i}=\left\{m \in \operatorname{Lie}(\mathfrak{X}) \mid \iota(a)(m)=\sigma^{-i}(a) m, \forall a \in \mathcal{O}_{d}\right\}
$$

où $\iota: \mathcal{O}_{D} \rightarrow \operatorname{End}(\mathfrak{X})$ exprime la structure de $\mathcal{O}_{D}$-module de $\mathfrak{X}$. Chaque $\operatorname{Lie}(\mathfrak{X})_{i}$ est un faisceau inversible sur $\widehat{\Omega}_{\breve{\mathcal{O}}}^{d-1}$. Considérons l'objet universel $(\boldsymbol{\psi}, \boldsymbol{\eta}, \mathbf{T}, \mathbf{u}, \mathbf{r})$ sur $\widehat{\Omega}_{\breve{\mathcal{O}}}^{d-1}$ rappelé dans (2.2.1). Dans la construction de l'isomorphisme de $G^{D r} \stackrel{\sim}{\longrightarrow} F^{D r}$ (voir [BC91, Théorème 8.4]), on identifie $\mathbf{T}_{i}$ à Lie $(\mathfrak{X})_{i}$, et l'action de $\Pi$ envoie $\mathbf{T}_{i}$ vers $\mathbf{T}_{i+1}$. On en déduit une décomposition de l'espace cotangent de $\mathfrak{X}[\Pi]$ :

$$
\operatorname{Lie}(\mathfrak{X}[\Pi])^{\vee}=\mathbf{T}_{0}^{\vee} / \Pi \mathbf{T}_{1}^{\vee} \oplus \mathbf{T}_{1}^{\vee} / \Pi \mathbf{T}_{2}^{\vee} \oplus \cdots \oplus \mathbf{T}_{d-1}^{\vee} / \Pi \mathbf{T}_{0}^{\vee} .
$$

Le morphisme $\iota$ induit un homomorphisme $\iota: \mathcal{O}_{D} / \Pi \simeq \mathbb{F}_{q^{d}} \rightarrow \operatorname{End}(\mathfrak{X}[\Pi])$. Ceci nous permet d'utiliser la classification de Raynaud [Ray74] que nous rappelons ci-dessous.

Soit $M=\operatorname{Hom}\left(\mathbb{F}_{q^{d}}^{\times}, \mathcal{O}_{D}^{\times}\right)$le groupe des caractères (homomorphisme de groupes) de $\mathbb{F}_{q^{d}}^{\times}$à valeurs dans $\mathcal{O}_{D}^{\times}$. On prolonge chaque caractère $\mu \in M$ à $\mathbb{F}_{q^{d}}=\mathcal{O}_{D} / \Pi \mathcal{O}_{D}$ tout entier en posant $\mu(0)=0$. Un caractère $\mu$ est dit fondamental si l'application composée $\mathbb{F}_{q^{d}} \stackrel{\mu}{\longrightarrow} \mathcal{O}_{D} \stackrel{\text { can }}{\longrightarrow} \mathbb{F}_{q^{d}}$ est un homomorphisme de corps. On a donc $f d$ caractères fondamentaux au total. Si on désigne $\chi: \mathbb{F}_{q^{d}}^{\times} \rightarrow \mathcal{O}_{d}^{\times} \subset \mathcal{O}_{D}^{\times}$le représentant de Teichmüller. Alors l'ensemble des caractères fondamentaux $\left\{\chi_{i}\right\}_{0 \leqslant i \leqslant f d-1}$ sont de la forme $\chi_{0}=\chi, \chi_{i}=\chi_{i-1}^{p}, 1 \leqslant i \leqslant f d-1$. Notons que $\bar{\iota}(\lambda)=\left.\iota(\chi(\lambda))\right|_{\mathfrak{X}[\Pi]} \in \operatorname{End}(\mathfrak{X}[\Pi])$, pour tout $\lambda \in \mathbb{F}_{q^{d}}$. 
Soit $\mathcal{O}_{\mathfrak{X}[\Pi]}=\mathcal{O}_{\widehat{\Omega}_{\breve{O}}^{d-1}} \oplus \mathcal{I}$, où $\mathcal{I}$ est l'idéal d'augmentation. L'endomorphisme $\bar{\iota}(\lambda)$ sur $\mathfrak{X}[\Pi]$ induit un endomorphisme $[\lambda]$ de l'algèbre de Hopf $\mathcal{O}_{\mathfrak{X}[\Pi]}$. Pour tout $\mu \in M$, les endomorphismes

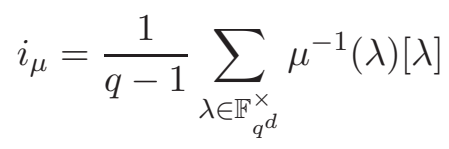

de la $\mathcal{O}_{\widehat{\Omega}_{\breve{\mathcal{O}}}^{d-1}}$-algèbre $\mathcal{O}_{\mathfrak{X}[\Pi]}$ forment une famille d'idempotents orthogonaux qui respectent $\mathcal{I}$. On a alors une décomposition

$$
\mathcal{I}=\bigoplus_{\mu \in M} \mathcal{I}_{\mu}
$$

où $\mathcal{I}_{\mu}=i_{\mu}(\mathcal{I})$ formé des éléments $x \in \mathcal{I}$ tels que $[\lambda](x)=\mu(\lambda) x$, pour tout $\lambda \in \mathbb{F}_{q^{d}}^{\times}$. Notons $\mathcal{I}_{i}:=\mathcal{I}_{\chi_{i}}, \forall 0 \leqslant i \leqslant f d-1$. Le $\widehat{\Omega}_{\breve{\mathcal{O}}}^{d-1}$-schéma en groupes $\mathfrak{X}[\Pi]$ satisfait la condition de la classification de Raynaud, i.e. chacun des faisceaux $\mathcal{I}_{i}$ est un $\mathcal{O}_{\widehat{\Omega}_{\breve{\mathcal{O}}}^{d-1}}$-module inversible, cf. [Ray74, Prop. 1.2.2]. Donc $\mathcal{I}$ est un $\mathcal{O}_{\widehat{\Omega}_{\tilde{\mathcal{O}}}^{d-1}}$-module localement libre de rang $q^{d}-1$.

(2.3.3) FAIT- ([Ray74, Thm. 1.4.1]) Sous ces conditions, le schéma en groupes $\mathfrak{X}[\Pi]$ est déterminé par le système $\left(\mathcal{I}_{i}, \bar{c}_{i}: \mathcal{I}_{i+1} \rightarrow \mathcal{I}_{i}^{p}, \bar{d}_{i}: \mathcal{I}_{i}^{p} \rightarrow \mathcal{I}_{i+1}\right)_{i}$ où les $\bar{c}_{i}$ et $\bar{d}_{i}$ sont $\mathcal{O}_{\widehat{\Omega}_{\tilde{\mathcal{O}}}^{d-1}}$-linéaires de sorte que $\bar{d}_{i} \circ \bar{c}_{i}=w \operatorname{Id}_{\mathcal{I}_{i+1}}, \forall 0 \leqslant i \leqslant f d-1$. Ici $w \in \Gamma\left(\widehat{\Omega}_{\breve{\mathcal{O}}}^{d-1}, \mathcal{O}_{\widehat{\Omega}_{\breve{\mathcal{O}}}^{d-1}}\right)$ est donné par la somme de Gauss indépendant du $\mathfrak{X}[\Pi]$.

Localement, on peut supposer que chaque $\mathcal{I}_{i}$ est en fait libre engendré par $X_{i}$. On en déduit que $\mathfrak{X}[\Pi]$ est donné localement sur $\widehat{\Omega}_{\breve{\mathcal{O}}}^{d-1}$ par les équations

$$
X_{i}^{p}=\delta_{i} X_{i+1}, i \in \mathbb{Z} / f d \mathbb{Z}
$$

avec $\delta_{i}$ des sections locales de $\widehat{\Omega}_{\breve{\mathcal{O}}}^{d-1}$. On a alors une autre description de l'espace cotangent de $\mathfrak{X}[\Pi]$ :

$$
\operatorname{Lie}(\mathfrak{X}[\Pi])^{\vee}=\mathcal{I} / \mathcal{I}^{2}=\mathcal{I}_{0} / \mathcal{I}_{f d-1}^{p} \oplus \mathcal{I}_{1} / \mathcal{I}_{0}^{p} \oplus \cdots \oplus \mathcal{I}_{f d-1} / \mathcal{I}_{f d-2}^{p}
$$

(2.3.5) Lemme.- $\mathbf{T}_{i}^{\vee} / \Pi \mathbf{T}_{i+1}^{\vee}=\left\{x \in \mathcal{I} / \mathcal{I}^{2} \mid[\lambda](x)=\sigma^{-i}(\chi(\lambda)) x, \forall \lambda \in \mathbb{F}_{q^{d}}\right\}$.

Preuve : On a $\operatorname{Lie}(\mathfrak{X}[\Pi])=\operatorname{Hom}\left(\mathcal{I} / \mathcal{I}^{2}, \mathcal{O}_{\widehat{\Omega}_{\tilde{\mathcal{O}}}^{d-1}}\right)$. Pour tout $\lambda \in \mathbb{F}_{q^{d}}, \iota(\lambda)$ induit une action $\operatorname{sur} \mathcal{I} / \mathcal{I}^{2}$ qui est, par définition, celle induite par $[\lambda]$.

En comparant les deux décompositions 2.3.2 et 2.3.4, on obtient que

$$
\mathcal{I}_{f i+j}=\mathcal{I}_{f i+j-1}^{p}, 1 \leqslant j \leqslant f-1 \text {, et } \mathcal{I}_{f i} / \mathcal{I}_{f i-1}^{p}=\mathbf{T}_{d-i}^{\vee} / \Pi \mathbf{T}_{d-i+1}^{\vee}
$$

On en déduit que

$$
\mathcal{I}_{f i} / \mathcal{I}_{f(i-1)}^{q}=\mathbf{T}_{d-i}^{\vee} / \Pi \mathbf{T}_{d-i+1}^{\vee}
$$

Il s'ensuit que $\mathfrak{X}[\Pi]$ est localement donné par les équations $X_{i}^{q}=\delta_{i} X_{i+1} \quad(i \in \mathbb{Z} / d \mathbb{Z})$. 
(2.3.6) Pour un simplexe $\sigma \in \mathcal{B T}$, on note $\widehat{\Omega}_{\breve{\mathcal{O}, \sigma}}^{d-1}$ le produit fibré de $\widehat{\Omega}_{\mathcal{O}, \sigma}^{d-1}$ avec $\widehat{\Omega}_{\breve{\mathcal{O}}}^{d-1}$ audessus de $\widehat{\Omega}_{\mathcal{O}}^{d-1}$, et $\Omega_{\breve{K}, \sigma}^{d-1}$ sa fibre générique. Si $\sigma=\Phi$ est le simplexe maximal standard, le morphisme $\Pi: \mathbf{T}_{i} \rightarrow \mathbf{T}_{i+1}$ est donné par multiplication par $c_{i}(c f$. (2.2.1) et (2.1.4)). Alors, $\operatorname{sur} \widehat{\Omega}_{\breve{\mathcal{O}, \Phi}}^{d-1}$

$$
\mathcal{I}_{f i} / \mathcal{I}_{f(i-1)}^{q}=\mathbf{T}_{d-i}^{\vee} / c_{d-i} \mathbf{T}_{d-i}^{\vee}
$$

(2.3.7) Lemme.- Soit s un sommet de $\mathcal{B} \mathcal{T}$. Notons $\Sigma_{s}$ l'espace rigide $\Sigma_{\Omega_{\breve{K}}^{d-1}} \Omega_{\breve{K}, s}^{d-1}$. Alors il existe une section $u \in \Gamma\left(\widehat{\Omega}_{\breve{\mathcal{O}}, s}^{d-1}, \mathcal{O}_{\widehat{\Omega}_{\breve{\mathcal{O}}, s}^{d-1}}^{*}\right)$ telle que

$$
\Sigma_{s} \cong \operatorname{Sp} \mathcal{O}_{\Omega_{\widetilde{K}, s}^{d-1}}\left[X_{0}\right] /\left(X_{0}^{q^{d}-1}-\varpi u\right)
$$

Preuve : Il suffit de traiter le cas où $s$ est le sommet standard $\Lambda=\left[\mathcal{O}^{d}\right]$. On observe tout d'abord que le groupe de Picard de $\widehat{\Omega}_{\breve{\mathcal{O}},[\Lambda]}^{d-1}$ est trivial. En effet, il est isomorphe au groupe de Picard de sa fibre spéciale, car $\widehat{\Omega}_{\breve{\mathcal{O}},[\Lambda]}^{d-1}$ est $\varpi$-adique complet (cf. [FvdP04, 3.7.4]). Donc

$$
\operatorname{Pic}\left(\widehat{\Omega}_{\breve{\mathcal{O},[\Lambda]}}^{d-1}\right)=\operatorname{Pic}\left(\Omega_{\overline{\mathbb{F}}_{q}}^{d-1}\right)=0,
$$

d'après [Har77, II Prop. 6.5]. Donc $\mathfrak{X}[\Pi] \times_{\widehat{\Omega}_{\mathscr{\mathcal { O }}}^{d-1}} \widehat{\Omega}_{\breve{\mathcal{O}},[\Lambda]}^{d-1}$ est donné par $X_{i}^{q}=\delta_{i} X_{i+1}$ où $\delta_{i} \in$ $\Gamma\left(\widehat{\Omega}_{\breve{\mathcal{O}},[\Lambda]}^{d-1}, \mathcal{O}_{\widehat{\Omega}_{\breve{\mathcal{O}},[\Lambda]}^{d-1}}\right)$. D'après l'exemple (2.1.4), il existe des sections $u_{i} \in \Gamma\left(\widehat{\Omega}_{\breve{\mathcal{O}},[\Lambda]}^{d-1}, \mathcal{O}_{\widehat{\Omega}_{\mathcal{O},[\Lambda]}^{d-1}}^{*}\right)$ telles que $\delta_{i}=c_{i} u_{i}, \forall i$. On en déduit que

$$
\begin{aligned}
\Sigma_{[\Lambda]} & =\operatorname{Sp} \mathcal{O}_{\Omega_{\breve{K},[\Lambda]}^{d-1}}\left[X_{0}\right] /\left(X_{0}^{q^{d}-1}-\delta_{0}^{q^{d-1}} \delta_{1}^{q^{d-2}} \cdots \delta_{d-1}\right) \\
& =\operatorname{Sp}_{\Omega_{\breve{K},[\Lambda]}^{d-1}}\left[X_{0}\right] /\left(X_{0}^{q^{d}-1}-c_{0} \cdots c_{d-1} u_{0}^{q^{d-1}} u_{1}^{q^{d-2}} \cdots u_{d-2}^{q} u_{d-1} c_{0}^{q^{d-1}-1} c_{1}^{q^{d-2}-1} \cdots c_{d-2}^{q-1}\right) \\
& =\operatorname{Sp} \mathcal{O}_{\Omega_{\breve{K},[\Lambda]}^{d-1}}\left[X_{0}\right] /\left(X_{0}^{q^{d}-1}-\varpi u\right),
\end{aligned}
$$

où $u:=u_{0}^{q^{d-1}} u_{1}^{q^{d-2}} \cdots u_{d-1} c_{0}^{q^{d-1}-1} c_{1}^{q^{d-2}-1} \cdots c_{d-2}^{q-1} \in \Gamma\left(\widehat{\Omega}_{\breve{\mathcal{O}},[\Lambda]}^{d-1}, \mathcal{O}_{\widehat{\Omega}_{\breve{\mathcal{O}},[\Lambda]}^{*}}^{*}\right)$, car $c_{0} \cdots c_{d-1}=\varpi$ et $c_{0}, \ldots, c_{d-2}$ appartiennent à $\Gamma\left(\widehat{\Omega}_{\breve{\mathcal{O}},[\Lambda]}^{d-1}, \mathcal{O}_{\widehat{\Omega}_{\breve{\mathcal{O}},[\Lambda]}^{d-1}}^{*}\right), c f$. l'exemple (2.1.4). D'où l'énoncé du lemme.

(2.3.8) Posons $\breve{K}^{t}=\breve{K}\left[\varpi_{t}\right] /\left(\varpi_{t}^{q^{d}-1}-\varpi\right)$ une extension modérément ramifiée de degré $q^{d}-1$ de $\breve{K}, \breve{\mathcal{O}}^{t}$ l'anneau des entiers de $\breve{K}^{t}$. Après l'extension des scalaires à $\breve{K}^{t}$, l'espace rigide $\Sigma_{s, \breve{K}^{t}}:=\Sigma_{s} \otimes_{\breve{K}} \breve{K}^{t}$ est donné par

$$
\operatorname{Sp} \mathcal{O}_{\Omega_{K^{t}, s}^{d-1}}\left[X_{0}^{\prime}\right] /\left(X_{0}^{\prime q^{d}-1}-u\right)
$$

où $X_{0}^{\prime}=X_{0} / \varpi_{t}$ et $\Omega_{\breve{K}^{t}, s}^{d-1}=\Omega_{\breve{K}, s}^{d-1} \otimes_{\breve{K}} \breve{K}^{t}$. Considérons la normalisation de $\widehat{\Omega}_{\breve{\mathcal{O}}, s}^{d-1}$ dans $\Sigma_{s, \breve{K}^{t}}$ que l'on notera $\widehat{\Sigma}_{s}^{0}$. D'après [SGA1, Exp. I (9.10), (10.2)], $\widehat{\Sigma}_{s}^{0}=\operatorname{Spf}_{\widehat{\Omega}_{\widetilde{O}^{t}, s}^{d-1}}\left[X_{0}^{\prime}\right] /\left(X_{0}^{\prime q^{d}-1}-u\right)$, avec $u \in \Gamma\left(\widehat{\Omega}_{\breve{\mathcal{O}}^{t}, s}^{d-1}, \mathcal{O}_{\widehat{\Omega}_{\mathcal{O}^{t}, s}^{d-1}}^{*}\right)$. Le groupe de Galois $\operatorname{Gal}\left(\breve{K}^{t} / \breve{K}\right)$ est isomorphe canoniquement à 
$\mu_{q^{d-1}}$ via $g \in \operatorname{Gal}\left(\breve{K}^{t} / \breve{K}\right) \mapsto g\left(\varpi_{t}\right) / \varpi_{t} \in \mu_{q^{d}-1}$. On en déduit qu'un élément $\zeta \in \mu_{q^{d-1}}$ agit sur l'anneau de schéma affine formel $\widehat{\Sigma}_{s}^{0}$ en envoyant $X_{0}^{\prime}$ vers $\zeta^{-1} X_{0}^{\prime}$.

Notons que la fibre speciale $\bar{\Sigma}_{s}^{0}$ de $\widehat{\Sigma}_{s}^{0}$ est un $\mu_{q^{d}-1^{-}}$-torseur $G_{s} / G_{s}^{+}$-invariant au-dessus de $\bar{\Omega}_{s}^{0}$, car $\bar{u}:=u\left(\bmod \varpi_{t}\right)$ est une unité dans $\Gamma\left(\bar{\Omega}_{s}^{0}, \mathcal{O}_{\bar{\Omega}_{s}^{0}}\right)$. On a alors un diagramme commutatif :

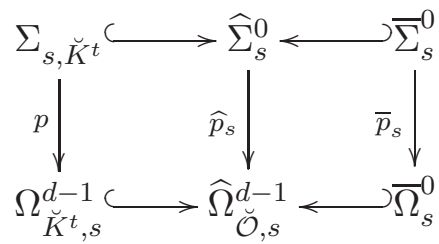

où $\widehat{p}$ et $\bar{p}$ désignent les projections naturelles.

(2.3.9) Corollaire.- On a un isomorphisme

$$
R \Gamma\left(\nu^{-1}(|s|), \Lambda\right) \cong R \Gamma\left(\bar{\Sigma}_{s}^{0}, \Lambda\right) .
$$

Preuve : D'après Berkovich, on a

$$
R \Gamma\left(\nu^{-1}(|s|), \Lambda\right)=R \Gamma\left(\tau^{-1}(|s|), p_{*} \Lambda\right)=R \Gamma\left(\bar{\Omega}_{s}^{0},\left.R \Psi_{\eta}\left(p_{*} \Lambda\right)\right|_{\bar{\Omega}_{s}^{0}}\right) .
$$

Notons que $\widehat{\Sigma}_{s}^{0}$ est un modèle lisse de $\Sigma_{s, \breve{K}^{t}}$. D'après [SGA7-1, Exp. I 2.4],

$$
\begin{aligned}
& \left.R^{0} \Psi_{\eta}\left(p_{*} \Lambda\right)\right|_{\bar{\Omega}_{s}^{0}}=\bar{p}_{s *} \Lambda, \\
& \left.R^{n} \Psi_{\eta}\left(p_{*} \Lambda\right)\right|_{\bar{\Omega}_{s}^{0}}=0, \forall n \geqslant 1 .
\end{aligned}
$$

Il s'ensuit que

$$
R \Gamma\left(\nu^{-1}(|s|), \Lambda\right)=R \Gamma\left(\bar{\Omega}_{s}^{0}, \bar{p}_{s *} \Lambda\right)=R \Gamma\left(\bar{\Sigma}_{s}^{0}, \Lambda\right) .
$$

\subsection{Un calcul du torseur $\bar{\Sigma}_{s}^{0}$}

Dans le paragraphe précédent, on a relié la cohomologie du tube $\nu^{-1}(|s|)$ à la cohomologie de $\bar{\Sigma}_{s}^{0}$. Rappelons que $\bar{\Sigma}_{s}^{0}$ est un $\mu_{q^{d}-1}$-torseur $G_{s} / G_{s}^{+}$-équivariant sur $\bar{\Omega}_{s}^{0}$. Dans ce paragraphe, notre but est de calculer sa classe dans $H_{e t}^{1}\left(\bar{\Omega}_{s}^{0}, \mu_{q^{d}-1}\right)$. Supposons désormais que $s$ soit le sommet standard $\Lambda=\left[\mathcal{O}^{d}\right]$. On se ramène donc au cas où $\bar{\Omega}_{s}^{0}=\Omega_{\overline{\mathbb{F}}_{q}}^{d-1}$ sur lequel $\bar{\Sigma}_{[\Lambda]}^{0}$ est un $\mu_{q^{d}-1}$-torseur $\mathrm{GL}_{d}\left(\mathbb{F}_{q}\right)$-équivariant.

(2.4.1) Notons $\mathcal{H}$ l'ensemble des hyperplans $\mathbb{F}_{q}$-rationnels de $\mathbb{P}_{\overline{\mathbb{F}}_{q}}^{d-1}$, nous avons alors

$$
\Omega_{\overline{\mathbb{F}}_{q}}^{d-1}=\mathbb{P}_{\overline{\mathbb{F}}_{q}}^{d-1} \backslash \bigcup_{Y \in \mathcal{H}} Y
$$

Notons $i$ (resp. $j$ ) l'inclusion naturelle de $D:=\bigcup_{Y \in \mathcal{H}} Y$ (resp. $\Omega_{\overline{\mathbb{F}}_{q}}^{d-1}$ ) dans $\mathbb{P}_{\overline{\mathbb{F}}_{q}}^{d-1}$. Pour $I$ un sous-ensemble de $\mathcal{H}$, on notera $Y_{I}=\bigcap_{Y \in I} Y$, et $i_{Y_{I}}$ l'inclusion de $Y_{I}$ dans $\mathbb{P}_{\overline{\mathbb{F}}_{q}}^{d-1}$. La suite exacte de cohomologie relative associée aux inclusions :

$$
\Omega_{\overline{\mathbb{F}}_{q}}^{d-1} \stackrel{j}{\longrightarrow} \mathbb{P}_{\overline{\mathbb{F}}_{q}}^{d-1} \stackrel{i}{\longleftarrow} \bigcup_{Y \in \mathcal{H}} Y
$$


nous fournit une suite exacte

$$
0 \longrightarrow H^{1}\left(\Omega_{\overline{\mathbb{F}}_{q}}^{d-1}, \mu_{n}\right) \stackrel{\partial}{\longrightarrow} H_{D}^{2}\left(\mathbb{P}_{\overline{\mathbb{F}}_{q}}^{d-1}, \mu_{n}\right) \longrightarrow H^{2}\left(\mathbb{P}_{\overline{\mathbb{F}}_{q}}^{d-1}, \mu_{n}\right)
$$

où $n$ est un entier premier à $p$.

(2.4.3) Lemme.- On a un isomorphisme canonique

$$
H_{D}^{2}\left(\mathbb{P}_{\overline{\mathbb{F}}_{q}}^{d-1}, \mu_{n}\right)=\bigoplus_{Y \in \mathcal{H}} H_{Y}^{2}\left(\mathbb{P}_{\overline{\mathbb{F}}_{q}}^{d-1}, \mu_{n}\right)=\bigoplus_{Y \in \mathcal{H}} \mathbb{Z} / n
$$

Preuve : On prend une résolution injective $\mathbb{Z} / n \rightarrow \mathcal{I}$ du faisceau constant $\mathbb{Z} / n$. Pour chaque $q$, nous avons une résolution simpliciale de $i_{*} i i^{\prime} \mathcal{I}^{q}$ :

$$
0 \rightarrow \cdots \rightarrow \bigoplus_{I \subset \mathcal{H},|I|=r} i_{Y_{I}, *} i_{Y_{I}}^{!}\left(\mathcal{I}^{q}\right) \rightarrow \cdots \rightarrow \bigoplus_{Y \in \mathcal{H}} i_{Y, *} i_{Y}^{!}\left(\mathcal{I}^{q}\right) \rightarrow i_{*} i^{!}\left(\mathcal{I}^{q}\right) \rightarrow 0
$$

Les suites spectrales associées à deux filtrations du double complexe $K^{p q}:=\bigoplus_{I \subset \mathcal{H},|I|=-p} i_{Y_{I}, *} i_{Y_{I}}^{!} \mathcal{I}^{q}(p \leqslant$ $-1, q \geqslant 0)$ nous fournit une suite spectrale :

$$
E_{1}^{p q}=\bigoplus_{I \subset \mathcal{H},|I|=-p} i_{Y_{I}, *} R^{q} i_{Y_{I}}^{!} \mathbb{Z} / n \Longrightarrow i_{*} R^{p+q} i ! \mathbb{Z} / n
$$

Pour chaque hyperplan rationnel $Y,\left(Y, \mathbb{P}_{\overline{\mathbb{F}}_{q}}^{d-1}\right)$ est un couple lisse ([SGA4-3, Exp. XVI] $)$ de codimension 1 , et $\left(Y_{I}, \mathbb{P}_{\overline{\mathbb{F}}_{q}}^{d-1}\right)$ est un couple lisse de codimension $>1$ si $|I| \geqslant 2$. D'après la pureté (voir loc. cit.), on sait que

$$
R^{0} i^{!}(\mathbb{Z} / n)=R^{1} i^{!}(\mathbb{Z} / n)=0
$$

et

$$
i_{*} R^{2} i^{!}(\mathbb{Z} / n)=\bigoplus_{Y \in \mathcal{H}} i_{Y *} R^{2} i_{Y}^{!}(\mathbb{Z} / n)=\bigoplus_{Y \in \mathcal{H}} i_{Y *}(\mathbb{Z} / n)_{Y}(-1) .
$$

On déduit la première égalité par la suite spectrale

$$
E_{2}^{p q}=H^{p}\left(D, R^{q} i^{!} \mu_{n}\right) \Longrightarrow H_{D}^{p+q}\left(\mathbb{P}_{\overline{\mathbb{F}}_{q}}^{d-1}, \mu_{n}\right)
$$

Chaque $Y$ est un diviseur irréductible, et la deuxième égalité est donnée par la classe fondamentale de $Y$ :

$$
H_{Y}^{2}\left(\mathbb{P}_{\mathbb{F}_{q}}^{d-1}, \mu_{n}\right)=H^{0}\left(Y, R^{2} i_{Y}^{!} \mu_{n}\right)=H^{0}\left(Y,(\mathbb{Z} / n)_{Y}\right)=\mathbb{Z} / n .
$$

(2.4.4) Posons $n=q^{d}-1$ et considérons la suite exacte 2.4.2. Reécrivons-la sous la forme suivante :

$$
0 \longrightarrow H_{e t}^{1}\left(\Omega_{\overline{\mathbb{F}}_{q}}^{d-1}, \mu_{q^{d}-1}\right) \stackrel{\partial}{\longrightarrow} \bigoplus_{Y \in \mathcal{H}} \mathbb{Z} /\left(q^{d}-1\right) \stackrel{\Sigma}{\longrightarrow} \mathbb{Z} /\left(q^{d}-1\right) .
$$

Notre but est de calculer la classe du $\mu_{q^{d}-1}$-torseur $\bar{\Sigma}_{[\Lambda]}^{0}$ dans $H_{e t}^{1}\left(\Omega_{\bar{F}_{q}}^{d-1}, \mu_{q^{d}-1}\right)$. Rappelons tout d'abord la définition de l'application $\partial$. Comme $\operatorname{Pic}\left(\Omega_{\overline{\mathbb{F}}_{q}}^{d-1}\right)=0$, par la suite exacte de Kummer, 
un $\mu_{q^{d}-1}$-torseur $Z$ peut être écrit sous la forme $\mathcal{O}_{\Omega_{\overline{\mathbb{F}}_{q}}^{d-1}}[T] /\left(T^{q^{d}-1}-f\right)$, avec $f \in \Gamma\left(\Omega_{\overline{\mathbb{F}}_{q}}^{d-1}, \mathcal{O}_{\Omega_{\mathbb{F}_{q}}^{d-1}}^{*}\right)$. Alors

$$
\partial(Z)(Y) \equiv \operatorname{ord}_{Y} f \quad\left(\bmod q^{d}-1\right), \forall Y \in \mathcal{H}
$$

(2.4.5) LeMme.- Soit $Z$ un $\mu_{q^{d}-1}$-torseur $\mathrm{GL}_{d}\left(\mathbb{F}_{q}\right)$-invariant sur $\Omega_{\mathbb{F}_{q}}^{d-1}$, alors $\partial(Z)(Y)=$ $\partial(Z)(g \cdot Y), \forall g \in \mathrm{GL}_{d}\left(\mathbb{F}_{q}\right)$, et $\partial(Z)(Y) \equiv 0(\bmod q-1)$.

Preuve : La première assertion découle d'invariance sous $g \in \mathrm{GL}_{d}\left(\mathbb{F}_{q}\right)$. Pour la deuxième, on observe que $\mathrm{GL}_{d}\left(\mathbb{F}_{q}\right)$ agit transitivement sur $\mathcal{H}$ et le cardinal de $\mathcal{H}$ est $1+q+q^{2}+\cdots+q^{d-1}$. Notons que $\partial(Z)$ est contenu dans le noyau de $\sum: \bigoplus_{Y \in \mathcal{H}} \mathbb{Z} /\left(q^{d}-1\right) \rightarrow \mathbb{Z} /\left(q^{d}-1\right)$, on a donc

$$
\left(1+q+\cdots+q^{d-1}\right) \cdot \partial(Z)(Y) \equiv 0 \quad\left(\bmod q^{d}-1\right) .
$$

Donc $\partial(Z)(Y) \equiv 0(\bmod q-1)$.

(2.4.6) ThÉORÈme.- Pour tout $Y \in \mathcal{H}$, on a $\partial\left(\bar{\Sigma}_{[\Lambda]}^{0}\right)(Y) \equiv q-1\left(\bmod q^{d}-1\right)$.

Preuve : Commençons par un lemme géométrique.

Lemme.- Soit $\sigma=\left\{\varpi \Lambda \subsetneq \Lambda_{0} \subsetneq \Lambda\right\}$ le simplexe que l'on a étudié dans l'exemple (2.1.4), i.e. $\Lambda_{0}$ correspond à l'hyperplan $\bar{x}_{0}=0$ de $\mathbb{P}(\Lambda / \varpi \Lambda)$, alors $\operatorname{Pic}\left(\widehat{\Omega}_{\breve{\mathcal{O}}, \sigma}^{d-1}\right)=0$.

Preuve : Il suffit de montrer que le groupe de Picard de la fibre spéciale $X$ de $\widehat{\Omega}_{\breve{\mathcal{O}, \sigma}}^{d-1}$ est triviale. D'après l'exemple (2.1.4), $X$ est une réunion des deux composantes irréductibles

$$
C=\operatorname{Spec} \overline{\mathbb{F}}_{q}\left[x_{0}, \ldots, x_{d-2}\right]\left[\prod\left(1+a_{0} x_{0}+\cdots+a_{d-2} x_{d-2}\right)^{-1}\right]
$$

et

$$
D=\operatorname{Spec} \overline{\mathbb{F}}_{q}\left[x_{1}, \ldots, x_{d-2}, c_{d-1}\right]\left[P^{-1}\right]
$$

où $P=\prod\left(1+a_{1} x_{1}+\cdots+a_{d-2} x_{d-2}\right)\left(1+a_{0} x_{1} c_{d-1}+\cdots+a_{d-3} x_{d-2} c_{d-1}+a_{d-2} c_{d-1}\right)$, avec l'intersection

$$
E:=C \cap D=\operatorname{Spec} \overline{\mathbb{F}}_{q}\left[x_{1}, \ldots, x_{d-2}\right]\left[\prod\left(1+a_{1} x_{1}+\cdots+a_{d-2} x_{d-2}\right)^{-1}\right] .
$$

Nous noterons $i_{C}, i_{D}$, et $i_{E}$ les inclusions canoniques dans $X$. D'après [Har77, II Prop. 6.5], $\operatorname{Pic}(C)=\operatorname{Pic}(D)=\operatorname{Pic}(E)=0$.

On montre qu'il existe une suite exacte des faisceaux sur $X$ :

$$
1 \longrightarrow \mathcal{O}_{X}^{*} \stackrel{\alpha}{\longrightarrow} i_{C *} \mathcal{O}_{C}^{*} \times i_{D *} \mathcal{O}_{D}^{*} \stackrel{\beta}{\longrightarrow} i_{E *} \mathcal{O}_{E}^{*} \longrightarrow 1
$$

où $\alpha$ est donné par $f \mapsto\left(\left.f\right|_{C},\left.f\right|_{D}\right), \beta$ est donné par $\left.\left.(f, g) \mapsto f\right|_{E} \cdot g\right|_{E} ^{-1}$. L'exactitude est vérifiée en regardant la fibre en chaque point. En effet, soit $x$ un point de $X$ contenu dans $C \backslash D$ (resp. $D \backslash C$ ), le complexe 2.4.7 se réduit à l'isomorphisme $\mathcal{O}_{X, x}^{*} \simeq \mathcal{O}_{C, x}^{*}\left(\operatorname{resp} . \mathcal{O}_{X, x}^{*} \simeq \mathcal{O}_{D, x}^{*}\right.$ ). Il nous reste le cas où $x$ est un point de $E$. Comme cette question est locale, on peut supposer que

$$
\begin{array}{rlrl}
X & =\operatorname{Spec} \overline{\mathbb{F}}_{q}\left[x_{0}, \ldots, x_{d-1}\right] / x_{0} x_{d-1} & C & =\operatorname{Spec} \overline{\mathbb{F}}_{q}\left[x_{0}, \ldots, x_{d-1}\right] / x_{0} \\
D & =\operatorname{Spec} \overline{\mathbb{F}}_{q}\left[x_{0}, \ldots, x_{d-1}\right] / x_{d-1} & E & =\operatorname{Spec} \overline{\mathbb{F}}_{q}\left[x_{0}, \ldots, x_{d-1}\right] /\left(x_{0}, x_{d-1}\right),
\end{array}
$$


et $x=\mathfrak{p}$ est un point de $E$. Notons que l'on a une suite exacte des faisceaux cohérents sur $X$

$$
0 \longrightarrow \mathcal{O}_{X} \stackrel{\alpha^{\prime}}{\longrightarrow} i_{C *} \mathcal{O}_{C} \times i_{D *} \mathcal{O}_{D} \stackrel{\beta^{\prime}}{\longrightarrow} i_{E *} \mathcal{O}_{E} \longrightarrow 0
$$

où $\alpha^{\prime}$ est donné par $f \mapsto\left(\left.f\right|_{C},\left.f\right|_{D}\right)$, $\beta^{\prime}$ est donné par $\left.(f, g) \mapsto f\right|_{E}-\left.g\right|_{E}$. On la vérifie en regardant les fibres en tous les points fermés. Donc on a une suite exacte

$$
0 \longrightarrow \mathcal{O}_{X, \mathfrak{p}} \stackrel{\alpha_{\mathfrak{p}}^{\prime}}{\longrightarrow} \mathcal{O}_{C, \mathfrak{p}} \times \mathcal{O}_{D, \mathfrak{p}} \stackrel{\beta_{\mathfrak{p}}^{\prime}}{\longrightarrow} \mathcal{O}_{E, \mathfrak{p}} \longrightarrow 0
$$

On en déduit que la suite

$$
1 \longrightarrow \mathcal{O}_{X, \mathfrak{p}}^{*} \stackrel{\alpha_{\mathfrak{p}}}{\longrightarrow} \mathcal{O}_{C, \mathfrak{p}}^{*} \times \mathcal{O}_{D, \mathfrak{p}}^{*} \stackrel{\beta_{\mathfrak{p}}}{\longrightarrow} \mathcal{O}_{E, \mathfrak{p}}^{*}
$$

est exacte. Notons que l'application surjective

$$
\overline{\mathbb{F}}_{q}\left[x_{0}, \ldots, x_{d-1}\right] / x_{d-1} \rightarrow \overline{\mathbb{F}}_{q}\left[x_{0}, \ldots, x_{d-1}\right] /\left(x_{0}, x_{d-1}\right)
$$

est scindée, et donc induit une surjection $\mathcal{O}_{D, \mathfrak{p}}^{*} \rightarrow \mathcal{O}_{E, \mathfrak{p}}^{*}$. C'est-à-dire $\beta_{\mathfrak{p}}$ est surjective.

On associe la suite exacte longue de cohomologie $H^{\bullet}(X,-)$ au complexe 2.4.7, et on obtient une suite exacte

$$
H^{0}\left(C, \mathcal{O}_{C}^{*}\right) \times H^{0}\left(D, \mathcal{O}_{D}^{*}\right) \stackrel{H^{0}(\beta)}{\longrightarrow} H^{0}\left(E, \mathcal{O}_{E}^{*}\right) \longrightarrow \operatorname{Pic}(X) \longrightarrow \operatorname{Pic}(C) \times \operatorname{Pic}(D) .
$$

L'immersion $E \hookrightarrow D$ est induite par l'épimorphisme

$$
\begin{aligned}
\varphi: \overline{\mathbb{F}}_{q}\left[x_{1}, \ldots, x_{d-2}, c_{d-1}\right]\left[P^{-1}\right] & \rightarrow \overline{\mathbb{F}}_{q}\left[x_{1}, \ldots, x_{d-2}\right]\left[\prod\left(1+a_{1} x_{1}+\cdots+a_{d-2} x_{d-2}\right)^{-1}\right] \\
x_{i} & \mapsto x_{i} \\
c_{d-1} & \mapsto 0 .
\end{aligned}
$$

Notons que $\varphi$ est scindé, donc il induit un épimorphisme $H^{0}\left(D, \mathcal{O}_{D}^{*}\right) \rightarrow H^{0}\left(E, \mathcal{O}_{E}^{*}\right)$. Alors $H^{0}(\beta)$ est surjectif, donc $\operatorname{Pic}(X)$ est trivial.

Revenons à la preuve du théorème. Rappelons que

$$
\Sigma_{[\Lambda]} \cong \operatorname{Sp} \mathcal{O}_{\Omega_{\breve{K},[\Lambda]}^{d-1}}[T] /\left(T^{q^{d}-1}-\varpi u\right)
$$

$c f$. le lemme (2.3.7). Grâce au lemme (2.4.5), il s'agit de calculer $\operatorname{ord}_{H} \bar{u}$, pour un hyperplan $\mathbb{F}_{q}$-rationnel $H$. On peut supposer que $H$ est l'hyperplan donné par $\bar{x}_{0}=0$. D'après le lemme précédent, $\mathfrak{X}[\Pi] \times_{\widehat{\Omega}_{\breve{\mathcal{O}}}^{d-1}} \widehat{\Omega}_{\breve{\mathcal{O}}, \sigma}^{d-1}$ est donné sur $\widehat{\Omega}_{\breve{\mathcal{O}}, \sigma}^{d-1}$ par les equations $X_{i}^{q}=\delta_{i} X_{i+1}$ où $\delta_{i} \in \Gamma\left(\widehat{\Omega}_{\breve{\mathcal{O}}, \sigma}^{d-1}, \mathcal{O}_{\widehat{\Omega}_{\breve{\mathcal{O}}, \sigma}^{d-1}}\right)$. En tenant compte de l'exemple (2.1.4), il est donné par

$$
\operatorname{Spf}_{\widehat{\Omega}_{\widetilde{\mathcal{O}}, \sigma}^{d-1}}\left[X_{0}, X_{1}\right] /\left(X_{0}^{q^{d-1}}-\delta_{1} X_{1}, X_{1}^{q}-\delta_{0} X_{0}\right)
$$

où $\delta_{1}=u_{1} x_{0}, \delta_{0}=u_{0} \varpi / x_{0}$, avec $u_{0}, u_{1} \in \Gamma\left(\widehat{\Omega}_{\breve{\mathcal{O}}, \sigma}^{d-1}, \mathcal{O}_{\widetilde{\Omega}_{\breve{\mathcal{O}}, \sigma}^{d-1}}^{*}\right)$. Ceci implique que $\Sigma_{\sigma}:=\Sigma \times_{\Omega_{\breve{K}}^{d-1}}$ $\Omega_{\breve{K}, \sigma}^{d-1}$ est l'espace rigide défini par

$$
\operatorname{Sp} \mathcal{O}_{\Omega_{\breve{K}, \sigma}^{d-1}}\left[X_{0}\right] /\left(X_{0}^{q^{d}-1}-\varpi u_{1}^{q} u_{0} x_{0}^{q-1}\right)
$$


Comme $\left.\Sigma_{\sigma}\right|_{\tau^{-1}(\Lambda)}$ et $\Sigma_{[\Lambda]}$ donnent la même classe de torseur dans $H^{1}\left(\Omega_{\breve{K},[\Lambda]}^{d-1}, \mu_{q^{d}-1}\right)$, il existe alors une fonction $f \in \Gamma\left(\Omega_{\breve{K},[\Lambda]}^{d-1}, \mathcal{O}_{\Omega_{\breve{K},[\Lambda]}^{d-1}}^{*}\right)$ telle que $\varpi u=\varpi u_{1}^{q} u_{0} x_{0}^{q-1} f^{q^{d}-1}$. Notons que $f$ appartient à $\Gamma\left(\widehat{\Omega}_{\breve{\mathcal{O}},[\Lambda]}^{d-1}, \mathcal{O}_{\widetilde{\Omega}_{\breve{\mathcal{O}},[\Lambda]}^{*-1}}^{*}\right)$, en fait $u, u_{0}, u_{1}, x_{0}$ sont tous dans $\Gamma\left(\widehat{\Omega}_{\breve{\mathcal{O}},[\Lambda]}^{d-1}, \mathcal{O}_{\widehat{\Omega}_{\breve{\mathcal{O}},[\Lambda]}^{d-1}}^{*}\right)$. Donc on a

$$
\operatorname{ord}_{\bar{x}_{0}} \bar{u} \equiv \operatorname{ord}_{\bar{x}_{0}} \bar{u}_{0}+\operatorname{ord}_{\bar{x}_{0}} \bar{u}_{1}^{q}+\operatorname{ord}_{\bar{x}_{0}} \bar{x}_{0}^{q-1} \quad\left(\bmod q^{d}-1\right) .
$$

Notons que $u_{0}, u_{1} \in \Gamma\left(\widehat{\Omega}_{\breve{\mathcal{O}}, \sigma}^{d-1}, \mathcal{O}_{\widehat{\Omega}_{\breve{\mathcal{O}}, \sigma}^{d-1}}^{*}\right)$, l'ordre de $\bar{u}_{i}$ en $\bar{x}_{0}$ est nulle. On en déduit que

$$
\operatorname{ord}_{\bar{x}_{0}} \bar{u} \equiv q-1 \quad\left(\bmod q^{d}-1\right)
$$

\subsection{Le lien avec les variétés de Deligne-Lusztig}

Considérons $\mathrm{GL}_{d}$ le groupe linéaire sur $\overline{\mathbb{F}}_{q}$ muni un morphisme de Frobenius $\left(a_{i, j}\right) \mapsto\left(a_{i, j}^{q}\right)$. Dans ce paragraphe, on rappelle certains aspects de la théorie de variétés de Deligne-Lusztig [DL76] associées à $\mathrm{GL}_{d}$ et l'élément de Coxeter $w:=(1, \ldots, d) \in \mathfrak{S}_{d}$, et on étudie le lien avec l'espace de Drinfeld $p$-adique.

(2.5.1) Les variétés de Deligne-Lusztig sont des variétés sur un corps fini $\mathbb{F}_{q}$, qui jouent un rôle important dans l'étude de la théorie de représentation de groupes finis réductifs. Ici on s'intéresse au cas associé à $\mathrm{GL}_{d}$ et l'élément de Coxeter $w:=(1, \ldots, d) \in \mathfrak{S}_{d}$, ceci est traité dans [DL76, (2.2)]. On a vu dans (2.4.1) que la sous-variété ouverte $\Omega_{\mathbb{F}_{q}}^{d-1}$ de $\mathbb{P}_{\mathbb{F}_{q}}^{d-1}$ est définie comme le complémentaire de tous les hyperplans $\mathbb{F}_{q}$-rationnels, donc elle est définie par la non-nullité du déterminant $\operatorname{det}\left(\left(X_{i}^{q^{j}}\right)_{0 \leqslant i, j \leqslant d-1}\right)$, car cette fonction s'identifie (à une constante non-nulle près) au produit de formes linéaires à coefficients dans $\mathbb{F}_{q}$, i.e.

$$
\operatorname{det}\left(\left(X_{i}^{q^{j}}\right)_{0 \leqslant i, j \leqslant d-1}\right)=c \cdot \prod_{\left[a_{0}: \cdots: a_{d-1}\right] \in \mathbb{P}^{d-1}\left(\mathbb{F}_{q}\right)}\left(a_{0} X_{0}+\cdots+a_{d-1} X_{d-1}\right),
$$

où $c \in \mathbb{F}_{q}^{\times}$dépend du choix des relèvements à $\mathbb{F}_{q}^{d} \backslash\{0\}$ des éléments de $\mathbb{P}^{d-1}\left(\mathbb{F}_{q}\right)$. Évidemment, le groupe fini $\mathrm{GL}_{d}\left(\mathbb{F}_{q}\right)$ agit sur $\Omega_{\mathbb{F}_{q}}^{d-1}$ par translation linéaire sur les coordonnées.

Par la construction de Deligne et Lusztig, $\Omega_{\mathbb{F}_{q}}^{d-1}$ admet un revêtement fini étale $\mathrm{DL}^{d-1}$ de groupe de Galois $\mathbb{F}_{q^{d}}^{\times}$. On peut identifier $\mathrm{DL}^{d-1}$ avec la sous-variété fermée de l'espace affine $\mathbb{A}_{\mathbb{F}_{q}}^{d}=\operatorname{Spec} \mathbb{F}_{q}\left[X_{0}, \ldots, X_{d-1}\right]$ définie par l'équation

$$
\operatorname{det}\left(\left(X_{i}^{q^{j}}\right)_{0 \leqslant i, j \leqslant d-1}\right)^{q-1}=(-1)^{d-1} .
$$

L'action de $\mathrm{GL}_{d}\left(\mathbb{F}_{q}\right)$ sur $\Omega_{\mathbb{F}_{q}}^{d-1}$ se lève naturellement sur $\mathrm{DL}^{d-1}$, et le groupe $\mathbb{F}_{q^{d}}^{\times}$agit sur DL ${ }^{d-1}$ par multiplication sur les coordonnées $X_{i} \mapsto \zeta X_{i}, \forall \zeta \in \mathbb{F}_{q^{d}}^{\times}$.

Posons $\mathrm{DL}_{\overline{\mathbb{F}}_{q}}^{d-1}:=\mathrm{DL}^{d-1} \otimes \overline{\mathbb{F}}_{q}$ le $\mu_{q^{d}-1^{-}}$-torseur $\mathrm{GL}_{d}\left(\mathbb{F}_{q}\right)$-invariant au-dessus de $\Omega_{\overline{\mathbb{F}}_{q}}^{d-1}$, et notons $\pi: \mathrm{DL}_{\overline{\mathbb{F}}_{q}}^{d-1} \rightarrow \Omega_{\overline{\mathbb{F}}_{q}}^{d-1}$ la projection canonique $\mathrm{GL}_{d}\left(\mathbb{F}_{q}\right)$-équivariante. Notons $x_{i}=X_{i} / X_{d-1}$, $0 \leqslant i \leqslant d-2$ les coordonnés affines associées, alors $\Omega_{\overline{\mathbb{F}}_{q}}^{d-1}$ s'identifie à $\mathbb{A}_{\overline{\mathbb{F}}_{q}}^{d-1}$ privé les hyperplans 
rationnels $a_{0} x_{0}+\cdots+a_{d-2} x_{d-2}+a_{d-1}$, où $\left(a_{0}, \ldots, a_{d-1}\right)$ parcourt $\mathbb{F}_{q}^{d} \backslash\{0\}$. De plus, nous avons une expression explicite du torseur $\mathrm{DL}_{\mathbb{F}_{q}}^{d-1}$ en posant $T:=1 / X_{d-1}$ :

$$
\begin{aligned}
\operatorname{DL}_{\bar{F}_{q}}^{d-1} & =\operatorname{Spec} \mathcal{O}_{\Omega_{\mathbb{F}_{q}-1}^{d-1}}[T] /\left(T^{q^{d}-1}-(-1)^{d-1}\left(\prod_{\left[a_{0}: \cdots: a_{d-1}\right] \in \mathbb{P}^{d-1}\left(\mathbb{F}_{q}\right)}\left(a_{0} x_{0}+\cdots+a_{d-2} x_{d-2}+a_{d-1}\right)\right)^{q-1}\right) \\
& \left.=\operatorname{Spec} \mathcal{O}_{\Omega_{\mathbb{F}_{q}}^{d-1}[T] /\left(T^{q^{d}-1}-(-1)^{d}\right.} \prod_{\left(a_{0}, \ldots, a_{d-1}\right) \in \mathbb{F}_{q}^{d} \backslash\{0\}}\left(a_{0} x_{0}+\cdots+a_{d-2} x_{d-2}+a_{d-1}\right)\right),
\end{aligned}
$$

$\operatorname{car} \prod_{\left(a_{0}, \ldots, a_{d-1}\right) \in \mathbb{F}_{q}^{d} \backslash\{0\}}\left(a_{0} x_{0}+\cdots+a_{d-2} x_{d-2}+a_{d-1}\right)=(-1) \cdot\left(\prod_{\left[a_{0}: \cdots: a_{d-1}\right] \in \mathbb{P}^{d-1}\left(\mathbb{F}_{q}\right)}\left(a_{0} x_{0}+\cdots+\right.\right.$ $\left.\left.a_{d-2} x_{d-2}+a_{d-1}\right)\right)^{q-1}$. On en déduit que $\zeta \in \mathbb{F}_{q^{d}}^{\times}$agit sur $\mathrm{DL}^{d-1}$ par la formule $T \mapsto \zeta^{-1} T$.

(2.5.3) Proposition.- Rappelons que $\mathcal{H}$ est l'ensemble des hyperplans $\mathbb{F}_{q}$-rationnels de $\mathbb{P}_{\mathbb{F}_{q}}^{d-1}$, cf. (2.4.1). Alors pour tout $Y \in \mathcal{H}$, on a $\partial\left(\mathrm{DL}_{\overline{\mathbb{F}}_{q}}^{d-1}\right)(Y) \equiv q-1\left(\bmod q^{d}-1\right)$.

Preuve : Le torseur $\mathrm{DL}_{\overline{\mathbb{F}}_{q}}^{d-1}$ est $\mathrm{GL}_{d}\left(\mathbb{F}_{q}\right)$-invariant. D'après le lemme (2.4.5), il suffit de savoir la valeur de $\partial\left(\mathrm{DL}_{\overline{\mathbb{F}}_{q}}^{d-1}\right)$ en l'hyperplan $X_{0}=0$ qui correspond à l'hyperplan $x_{0}=0$ de $\mathbb{A}_{\mathbb{F}_{q}}^{d-1}$. Alors,

$$
\begin{aligned}
\partial\left(\mathrm{DL}_{\overline{\mathbb{F}}_{q}}^{d-1}\right)\left(X_{0}=0\right) & =\operatorname{ord}_{x_{0}}\left((-1)^{d} \cdot \prod_{\left(a_{0}, \ldots, a_{d-1}\right) \in \mathbb{F}_{q}^{d} \backslash\{0\}}\left(a_{0} x_{0}+\cdots+a_{d-2} x_{d-2}+a_{d-1}\right)\right) \\
& \equiv q-1 \quad\left(\bmod q^{d}-1\right) .
\end{aligned}
$$

(2.5.4) ThÉorème.- Soit s un sommet de $\mathcal{B T}$. Quitte à choisir une base d'un réseau qui représente $s$, on a un isomorphisme $G_{s} / G_{s}^{+} \cong \mathrm{GL}_{d}\left(\mathbb{F}_{q}\right)$-équivariant

$$
R \Gamma\left(\nu^{-1}(|s|), \Lambda\right) \stackrel{\sim}{\longrightarrow} R \Gamma\left(\mathrm{DL}_{\overline{\mathbb{F}}_{q}}^{d-1}, \Lambda\right) .
$$

Preuve : D'après le théorème (2.4.6) et la proposition (2.5.3), on a un isomorphisme $G_{s} / G_{s}^{+} \cong \mathrm{GL}_{d}\left(\mathbb{F}_{q}\right)$-équivariant $\bar{\Sigma}_{s}^{0} \cong \mathrm{DL}_{\overline{\mathbb{F}}_{q}}^{d-1}$ et un diagramme commutatif :

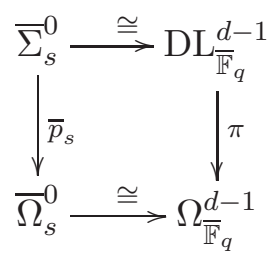

En vertu du corollaire (2.3.9), on obtient un isomorphisme

$$
R \Gamma\left(\nu^{-1}(|s|), \Lambda\right) \stackrel{\sim}{\longrightarrow} R \Gamma\left(\mathrm{DL}_{\overline{\mathbb{F}}_{q}}^{d-1}, \Lambda\right) .
$$

(2.5.6) Corollaire.- On a un isomorphisme $G_{s} / G_{s}^{+} \cong \mathrm{GL}_{d}\left(\mathbb{F}_{q}\right)$-équivariant :

$$
R \Gamma_{c}\left(\nu^{-1}\left(|s|^{*}\right), \Lambda\right) \stackrel{\sim}{\longrightarrow} R \Gamma_{c}\left(\mathrm{DL}_{\overline{\mathbb{F}}_{q}}^{d-1}, \Lambda\right) .
$$


Preuve: Ceci découle du théorème (2.2.3) et le théorème précédent, en vertu de la dualité de Poincaré analytique [Ber93, (7.3)] et algébrique [SGA4-3, Exp. XVIII].

(2.5.7) Dans [DL76, §9], Deligne et Lusztig ont introduit une compactification $\bar{\Omega}_{\mathbb{F}_{q}}^{d-1}$ de $\Omega_{\mathbb{F}_{q}}^{d-1}$ dont le complémentaire est un diviseur à croisements normaux. Soit $\Delta=\left\{\alpha_{1}, \ldots, \alpha_{d-1}\right\}$ l'ensemble de racines simples tel que $w=s_{\alpha_{1}} \cdots s_{\alpha_{d-1}} \in \mathfrak{S}_{d}$, suivant eux, on définit

$$
\bar{\Omega}_{\mathbb{F}_{q}}^{d-1}:=\coprod_{\substack{x=x_{1} \cdots x_{d-1} \in W \\ x_{i} \in\left\{1, s_{\alpha_{i}}\right\}}} X(x),
$$

où $X(x)$ est la variété de Deligne-Lusztig associée à $\mathrm{GL}_{d}$ et l'élément $x \in \mathfrak{S}_{d}$, en particulier, $X(w)=\Omega_{\mathbb{F}_{q}}^{d-1}$. D'ailleurs, la variété $\bar{\Omega}_{\mathbb{F}_{q}}^{d-1}$ peut s'obtenir par une suite d'éclatements successifs de l'espace projectif $\mathbb{P}_{\mathbb{F}_{q}}^{d-1}$ comme dans (2.1.5), cf. [Wan13, Lemme 4.1.2].

FAIT.- Soit $s$ un sommet de $\mathcal{B} \mathcal{T}$. Quitte à choisir une base d'un réseau qui représente $s$, on a un diagramme commutatif $G_{s} / G_{s}^{+} \cong \mathrm{GL}_{d}\left(\mathbb{F}_{q}\right)$-équivariant :

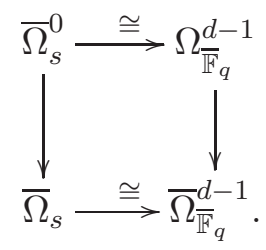

Preuve: Ceci découle de [Wan13, Lemme 4.1.2].

Remarque.- Soient $I$ un sous-ensemble de $\Delta, P_{I}=B W_{I} B$ le sous-groupe parabolique standard associé à $I$ et $U_{I}$ le radical unipotent de $P_{I}$. Dès que l'on fait un choix d'un sommet $s \in \mathcal{B} \mathcal{T}_{0}$ et une base d'un réseau qui représente $s$, l'isomorphisme dans le fait précédent nous fournit un sous-groupe parabolique de $G_{s} / G_{s}^{+}$, et donc un simplexe $\sigma$ contenant $s$. De plus, on a un diagramme commutatif :

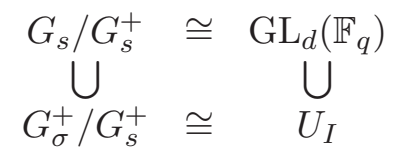

FAIT.- Sous l'hypothèse du fait précédent, l'isomorphisme $\bar{\Omega}_{s} \stackrel{\sim}{\longrightarrow} \bar{\Omega}_{\overline{\mathbb{F}}_{q}}^{d-1}$ comme dans loc. cit. identifie $\bar{\Omega}_{\sigma}$ à $\bar{C}_{I}$, où $\bar{C}_{I}:=\left(\bar{\Omega}_{\mathbb{F}_{q}}^{d-1}\right)^{U_{I}}$ les points stables sous $U_{I}$.

Preuve : Supposons tout d'abord que $I=\Delta \backslash\left\{\alpha_{i}\right\}$ pour une racine simple $\alpha_{i} \in \Delta$. D'après ce qui précède, $I$ fait un choix d'un simplexe $\left[s, s^{\prime}\right]$ contenant $s$, où $s^{\prime}$ est un sommet voisin de $s$. La remarque dans [Wan13, (4.1.4)] nous dit que $\bar{C}_{I}$ s'identifie à $\bar{\Omega}_{s} \cap \bar{\Omega}_{s^{\prime}}=\bar{\Omega}_{\left[s, s^{\prime}\right]}$. Pour $I$ quelconque, supposons que $I=\Delta \backslash\left\{\alpha_{i_{1}}, \ldots, \alpha_{i_{r}}\right\}$. Alors $I=I_{1} \cap \cdots \cap I_{r}$, où $I_{j}=\Delta \backslash\left\{\alpha_{i_{j}}\right\}$, et $\bar{C}_{I}=\bar{C}_{I_{1}} \cap \cdots \cap \bar{C}_{I_{r}}$. Chaque $I_{j}$ fait un choix d'un sommet voisin $s_{j}$ de $s$, et identifie $\bar{C}_{I_{j}}$ à $\bar{\Omega}_{\left[s, s_{j}\right]}$. Notons que $\sigma:=\left[s, s_{1}, \ldots, s_{r}\right]$ est le simplexe donné par $I$. Par définition, $\bar{\Omega}_{\sigma}=$ $\bar{\Omega}_{\left[s, s_{1}\right]} \cap \cdots \cap \bar{\Omega}_{\left[s, s_{r}\right]}$, donc il s'identifie à $\bar{C}_{I}$. 
(2.5.8) Rappelons le théorème principal (Théorème 5.1.1) de [Wan13]. Considérons le diagramme suivant :

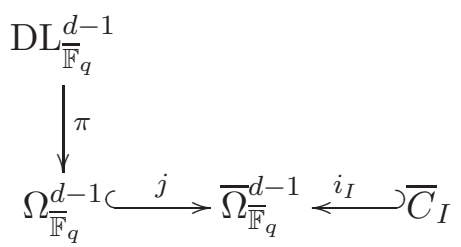

THÉORÈME.- Le morphisme de restriction

$$
R \Gamma\left(\Omega_{\overline{\mathbb{F}}_{q}}^{d-1}, \pi_{*} \Lambda\right)=R \Gamma\left(\bar{\Omega}_{\overline{\mathbb{F}}_{q}}^{d-1}, R j_{*}\left(\pi_{*} \Lambda\right)\right) \stackrel{\text { res }}{\longrightarrow} R \Gamma\left(\bar{C}_{I}, i_{I}^{*} R j_{*}\left(\pi_{*} \Lambda\right)\right)
$$

induit un isomorphisme

$$
R \Gamma\left(\Omega_{\overline{\mathbb{F}}_{q}}^{d-1}, \pi_{*} \Lambda\right)^{U_{I}} \stackrel{\sim}{\longrightarrow} R \Gamma\left(\bar{C}_{I}, i_{I}^{*} R j_{*}\left(\pi_{*} \Lambda\right)\right) .
$$

Cela nous donne la conséquence suivante sur la cohomologie de $\Sigma^{c a}$.

(2.5.9) ThÉorème.- Soient $s$ un sommet de $\mathcal{B} \mathcal{T}$ et $\sigma$ un simplexe contenant $s$, alors le morphisme canonique $H_{c}^{q}\left(\nu^{-1}\left(|\sigma|^{*}\right), \Lambda\right) \rightarrow H_{c}^{q}\left(\nu^{-1}\left(|s|^{*}\right), \Lambda\right)$ provenant de l'immersion ouverte $\nu^{-1}\left(|\sigma|^{*}\right) \hookrightarrow \nu^{-1}\left(|s|^{*}\right)$ induit un isomorphisme

$$
H_{c}^{q}\left(\nu^{-1}\left(|\sigma|^{*}\right), \Lambda\right) \stackrel{\sim}{\longrightarrow} H_{c}^{q}\left(\nu^{-1}\left(|s|^{*}\right), \Lambda\right)^{G_{\sigma}^{+}} .
$$

Preuve: Par la dualité de Poincaré, il suffit de montrer que le morphisme de restriction $R \Gamma\left(\nu^{-1}\left(|s|^{*}\right), \Lambda\right) \longrightarrow R \Gamma\left(\nu^{-1}\left(|\sigma|^{*}\right), \Lambda\right)$ induit un ismorphisme $R \Gamma\left(\nu^{-1}\left(|s|^{*}\right), \Lambda\right)_{G_{\sigma}^{+}} \stackrel{\sim}{\longrightarrow}$ $R \Gamma\left(\nu^{-1}\left(|\sigma|^{*}\right), \Lambda\right)$. D'après Berkovich [Ber96, Corollary 3.5],

$$
R \Gamma\left(\nu^{-1}\left(|\sigma|^{*}\right), \Lambda\right)=R \Gamma\left(\bar{\Omega}_{\sigma},\left.R \Psi_{\eta}\left(p_{*} \Lambda\right)\right|_{\bar{\Omega}_{\sigma}}\right),
$$

où $p$ est la projection $\Sigma^{c a} \rightarrow \Omega_{K}^{d-1, c a}$.

Notons $\bar{p}_{s}: \bar{\Sigma}_{s}^{0} \rightarrow \bar{\Omega}_{s}^{0}$ et $i_{\sigma}: \bar{\Omega}_{\sigma} \hookrightarrow \bar{\Omega}_{s}$. D'après le théorème (2.2.3) et le corollaire (2.3.9), on a un diagramme commutatif :

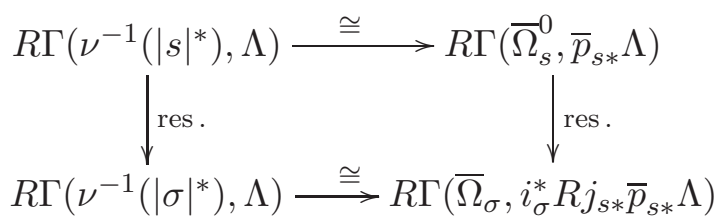

Donc on se ramène à montrer que le morphisme de restriction induit un isomorphisme

$$
R \Gamma\left(\bar{\Omega}_{s}^{0}, \bar{p}_{s *} \Lambda\right)_{G_{\sigma}^{+} / G_{s}^{+}} \stackrel{\sim}{\longrightarrow} R \Gamma\left(\bar{\Omega}_{\sigma}, i_{\sigma}^{*} R j_{s, *} \bar{p}_{s *} \Lambda\right) .
$$

Comme $G_{\sigma}^{+} / G_{s}^{+}$est un $p$-groupe fini et $\Lambda$ est de torsion premier à $p$, on peut identifier canoniquement $R \Gamma\left(\bar{\Omega}_{s}^{0}, \bar{p}_{s *} \Lambda\right)_{G_{\sigma}^{+} / G_{s}^{+}}$à $R \Gamma\left(\bar{\Omega}_{s}^{0}, \bar{p}_{s *} \Lambda\right)^{G_{\sigma}^{+} / G_{s}^{+}}$. On obtient l'énoncé du théorème, en vertu du théorème précédent et les isomorphismes canoniques :

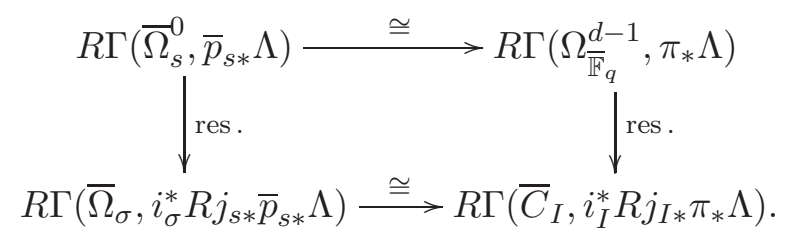




\section{La partie supercuspidale de la cohomologie}

Dans cette section, on donne quelques conséquences sur la partie supercuspidale de la cohomologie de $\Sigma^{c a}$.

\subsection{La démonstration de Théorème $\mathrm{A}$.}

Fixons $\ell \neq p$ un nombre premier et une clôture algébrique $\overline{\mathbb{Q}}_{\ell}$ de $\mathbb{Q}_{\ell}$.

(3.1.1) Soient $\sigma^{\prime} \subset \sigma$ deux simplexes de $\mathcal{B} \mathcal{T}$, l'immersion ouverte $\nu^{-1}\left(|\sigma|^{*}\right) \hookrightarrow \nu^{-1}\left(\left|\sigma^{\prime}\right|^{*}\right)$ induit un morphisme canonique :

$$
H_{c}^{q}\left(\nu^{-1}\left(|\sigma|^{*}\right), \Lambda\right) \longrightarrow H_{c}^{q}\left(\nu^{-1}\left(\left|\sigma^{\prime}\right|^{*}\right), \Lambda\right) .
$$

Donc les données

$$
\left\{\begin{array}{l}
(\sigma \in \mathcal{B T}) \mapsto H_{c}^{q}\left(\nu^{-1}\left(|\sigma|^{*}\right), \Lambda\right) \\
\left(\sigma^{\prime} \subset \sigma\right) \mapsto\left(H_{c}^{q}\left(\nu^{-1}\left(|\sigma|^{*}\right), \Lambda\right) \rightarrow H_{c}^{q}\left(\nu^{-1}\left(\left|\sigma^{\prime}\right|^{*}\right), \Lambda\right)\right)
\end{array}\right.
$$

définissent un système de coefficients $G^{\circ}$-équivariant (voir [Dat06, 3.2.3]) à valeurs dans les $\Lambda$-modules que nous noterons simplement $\sigma \mapsto H_{c}^{q}\left(\nu^{-1}\left(|\sigma|^{*}\right), \Lambda\right)$. Nous avons le fait suivant bien connu (voir [Dat06, Prop. 3.2.4] pour le fait et [Dat06, 3.2.3] pour les notations) :

FAIT.- Il existe une suite spectrale $G^{\circ}$-équivariante

$$
E_{1}^{p q}=\mathcal{C}_{c}^{o r}\left(\mathcal{B T}_{(-p)}, \sigma \mapsto H_{c}^{q}\left(\nu^{-1}\left(|\sigma|^{*}\right), \Lambda\right)\right) \Longrightarrow H_{c}^{p+q}\left(\Sigma^{c a}, \Lambda\right)
$$

dont la différentielle $d_{1}^{p q}$ est celle du complexe de chaînes du système de coefficients $\sigma \mapsto$ $H_{c}^{q}\left(\nu^{-1}\left(|\sigma|^{*}\right), \Lambda\right)$.

(3.1.3) Notons $W_{K}$ le groupe de Weil associé à $K$ et $I_{K}$ le sous-groupe d'inertie. Posons $G D W:=G \times D^{\times} \times W_{K}$, et $v: G D W \rightarrow \mathbb{Z}$ l'homorphisme qui envoie un élément $(g, \delta, w)$ vers l'entier $\operatorname{val}_{K}\left(\operatorname{det}\left(g^{-1}\right) \operatorname{Nr}(\delta) \operatorname{Art}^{-1}(w)\right) \in \mathbb{Z}$, où $\mathrm{Nr}: D^{\times} \rightarrow K^{\times}$désigne la norme réduite et Art $^{-1}: W_{K} \rightarrow W_{K}^{a b} \rightarrow K^{\times}$désigne la composée de l'inverse du morphisme d'Artin qui envoie l'uniformisante $\varpi$ vers un Frobenius géométrique fixé $\varphi$. On désigne $(G D W)^{0}:=v^{-1}(0)$ le noyau de $v$, et $[G D W]_{d}$ le sous-groupe distingué formé des éléments $(g, \delta, w)$ tels que $v(g, \delta, w) \in$ $d \mathbb{Z}$. On considère $G$ (resp. $D^{\times}, W_{K}$ ) comme un sous-groupe de $G D W$ via l'inclusion naturelle, et on notera $[G]_{d}$ (resp. $[D]_{d},\left[W_{K}\right]_{d}$ ) son intersection avec $[G D W]_{d}$. Dès que l'on identifie $K^{\times}$ au centre de $G$, on a $[G D W]_{d}=(G D W)^{0} \varpi^{\mathbb{Z}}$.

(3.1.4) On a vu dans le paragraphe 2.2 que l'espace analytique $\Sigma^{c a}$ admet une action naturelle du groupe $G^{\circ} \times \mathcal{O}_{D}^{\times} \times I_{K}$. Afin de définir une action du groupe $G D W$, on suit la construction de Rapoport-Zink [RZ96] en adoptant les notations de [Dat07, §3], i.e. on ne fixe pas la hauteur des quasi-isogénies qui rigidifient les problèmes de modules dans la définition du foncteur $G^{D r}$ dans (2.2.1). Ce nouveau problème de modules $\widetilde{G}$ est de même pro-représentable

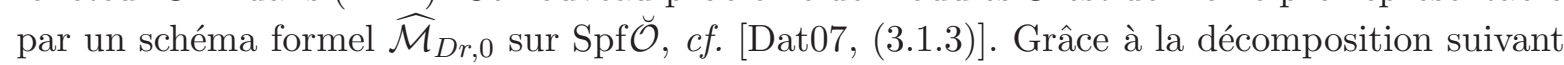
la hauteur de quasi-isogénie, $\widetilde{G}=\coprod_{h \in \mathbb{Z}} G^{(h)}$ où $G^{(h)}$ classifie les classes de triples $(\psi, X, \rho)$ avec $\rho$ de hauteur $d h$. Chaque $G^{(h)}$ est isomorphe (non-canoniquement) à $G^{(0)}$ et $G^{(0)}$ est le foncteur $G^{D r}$ de Drinfeld. On en déduit un isomorphisme non-canonique $\widehat{\mathcal{M}}_{D r, 0} \cong \widehat{\mathcal{M}}_{D r, 0}^{(0)} \times \mathbb{Z}$ 
où $\widehat{\mathcal{M}}_{D r, 0}^{(0)}:=\widehat{\Omega}_{\breve{\mathcal{O}}}^{d-1}$ est le schéma formel qui représente $G^{D r}$. On note $\widetilde{\mathfrak{X}}$ le $\mathcal{O}_{D}$-module formel spécial universel sur $\widehat{\mathcal{M}}_{D r, 0}$. Le noyau $\widetilde{\mathfrak{X}}\left[\Pi_{D}\right]$ de la multiplication par $\Pi_{D}$ dans $\widetilde{\mathfrak{X}}$ est un schéma formel en groupe fini plat de rang $q^{d}$ au-dessus de $\widehat{\mathcal{M}}_{D r, 0}$, et qui est étale en fibre générique. Le $\left(\mathcal{O}_{D} / \Pi_{D} \mathcal{O}_{D}\right)^{\times}$-torseur $\underline{\operatorname{Isom}}_{\mathcal{O}_{D}}\left(\mathcal{O}_{D} / \Pi_{D} \mathcal{O}_{D}, \widetilde{\mathfrak{X}}\left[\Pi_{D}\right]^{a n}\right)$ sur $\mathcal{M}_{D r, 0}$ la fibre générique au sens de Raynaud-Berkovich de $\widehat{\mathcal{M}}_{D r, 0}$, est donc représenté par un $\breve{K}$-espace analytique $\mathcal{M}_{D r, 1}$, qui est un revêtement étale de $\mathcal{M}_{D r, 0}$ de groupe $\mathcal{O}_{D}^{\times} /\left(1+\Pi_{D} \mathcal{O}_{D}\right) \cong \mathbb{F}_{q^{d}}^{\times}$. En posant $\mathcal{M}_{D r, 1}^{c a}:=$ $\mathcal{M}_{D r, 1} \widehat{\otimes}_{\breve{K}} \widehat{K^{c a}}$, on a un isomorphisme $\mathcal{M}_{D r, 1}^{c a} \cong \Sigma^{c a} \times \mathbb{Z}$. Suivant [Dat07, (3.1.5)], on définit une action du groupe $G D W$ sur $\mathcal{M}_{D r, 1}^{c a}$ telle que la composante $\Sigma^{c a} \times\{0\}$ soit stable sous l'action du sous-groupe $(G D W)^{0}$. Désormais, on identifie $\Sigma^{c a}$ au sous-espace analytique ouvert-fermé $\Sigma^{c a} \times\{0\}$ de $\mathcal{M}_{D r, 1}^{c a}$.

(3.1.5) Soit $\theta: \mathbb{F}_{q^{d}}^{\times} \rightarrow \overline{\mathbb{Q}}_{\ell}^{\times}$un caractère $d$-primitif, i.e. $\theta$ ne se factorise pas par la norme

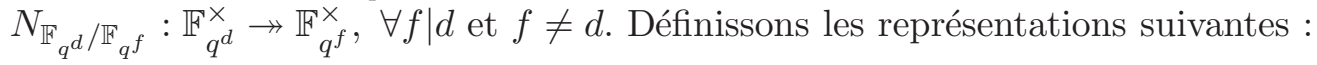

- une représentation $\rho(\theta):=\operatorname{ind}_{[D]_{d}}^{D^{\times}} \theta$ de $D^{\times}$, où $[D]_{d}=\mathcal{O}_{D}^{\times} \varpi^{\mathbb{Z}}$ tel que $\mathcal{O}_{D}^{\times}$agit via la projection $\mathcal{O}_{D}^{\times} \rightarrow \mathbb{F}_{q^{d}}^{\times}$, et $\varpi^{\mathbb{Z}}$ agit trivialement.

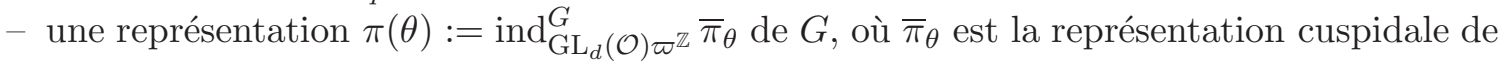
$\mathrm{GL}_{d}\left(\mathbb{F}_{q}\right)$ associée à $\theta$ via la correspondance de Green (ou de Deligne-Lusztig), vue comme une représentation de $\mathrm{GL}_{d}(\mathcal{O}) \varpi^{\mathbb{Z}}$ telle que $\mathrm{GL}_{d}(\mathcal{O})$ agit via la projection $\mathrm{GL}_{d}(\mathcal{O}) \rightarrow$ $\mathrm{GL}_{d}\left(\mathbb{F}_{q}\right)$ et $\varpi^{\mathbb{Z}}$ agit trivialement.

- une représentation $\sigma^{\sharp}(\theta):=\operatorname{ind}_{\left[W_{K}\right]_{d}}^{W_{K}} \widetilde{\theta}$ de $W_{K}$, où $\widetilde{\theta}$ est le caractère de $\left[W_{K}\right]_{d}=I_{K}\left\langle\varphi^{d}\right\rangle^{\mathbb{Z}}$ tel que $\widetilde{\theta}\left(\varphi^{d}\right)=(-1)^{d-1} q^{\frac{d(d-1)}{2}}$, et $\left.\widetilde{\theta}\right|_{I_{K}}$ se factorise par l'inertie modérée $I_{K} \rightarrow I_{K} / I_{K\left(\varpi_{t}\right)} \cong$ $\mu_{q^{d}-1} \stackrel{\theta}{\longrightarrow} \overline{\mathbb{Q}}_{\ell}^{\times}$, ici $\varpi_{t}$ est la racine $\left(q^{d}-1\right)$-ième de $\varpi$ fixée dans $(2.3 .8)$.

Posons

$$
\mathbf{H}_{c}^{i}:=H_{c}^{i}\left(\mathcal{M}_{D r, 1}^{c a} / \varpi^{\mathbb{Z}}, \overline{\mathbb{Q}}_{\ell}\right), i \in\{d-1, \ldots, 2 d-2\} .
$$

D'après la description précédente,

$$
\mathbf{H}_{c}^{i}=\operatorname{ind}_{[G D W]_{d}}^{G D W} H_{c}^{i}\left(\Sigma^{c a}, \overline{\mathbb{Q}}_{\ell}\right),
$$

où $\varpi \in K^{\times} \subset G$ agit trivialement. On considère la partie $\rho(\theta)$-isotypique de $\mathbf{H}_{c}^{i}$.

(3.1.6) ThÉORÈme.- Pour tout $\theta: \mathbb{F}_{q^{d}}^{\times} \rightarrow \overline{\mathbb{Q}}_{\ell}^{\times}$caractère d-primitif, on a

$$
\operatorname{Hom}_{D^{\times}}\left(\rho(\theta), \mathbf{H}_{c}^{i}\right) \underset{G \times \overline{\widetilde{W}}_{K}}{\simeq} \begin{cases}\pi(\theta) \otimes \sigma^{\sharp}(\theta), & \text { si } i=d-1 ; \\ 0, & \text { si } i \neq d-1 .\end{cases}
$$

Preuve : Ceci découle du lemme (3.1.8) et la proposition (3.1.10).

(3.1.7) Lemme.- En tant que représentation de $G W:=G \times W_{K}$, on a

$$
\operatorname{Hom}_{D^{\times}}\left(\rho(\theta), \mathbf{H}_{c}^{i}\right)=\operatorname{ind}_{[G W]_{d}}^{G W} \operatorname{Hom}_{\mathbb{F}_{q^{d}}^{\times}}\left(\theta, H_{c}^{i}\left(\Sigma^{c a}, \overline{\mathbb{Q}}_{\ell}\right)\right),
$$

ò̀ $[G W]_{d}=\{(g, w) \in G W \mid v(g, 1, w) \in d \mathbb{Z}\}$. 
Preuve: D'après la réciprocité de Frobenius, on a

$$
\begin{aligned}
\operatorname{Hom}_{D^{\times}}\left(\rho(\theta), \mathbf{H}_{c}^{i}\right) & =\operatorname{Hom}_{[D]_{d}}\left(\theta, \operatorname{ind}_{[G D W]_{d}}^{G D W} H_{c}^{i}\left(\Sigma^{c a}, \overline{\mathbb{Q}}_{\ell}\right)\right) \\
& =\operatorname{ind}_{[G W]_{d}}^{G W} \operatorname{Hom}_{\mathbb{F}_{q^{d}}^{\times}}\left(\theta, H_{c}^{i}\left(\Sigma^{c a}, \overline{\mathbb{Q}}_{\ell}\right)\right) .
\end{aligned}
$$

(3.1.8) Lemme.- En tant que représentation de $G$, on a

$$
\operatorname{Hom}_{D^{\times}}\left(\rho(\theta), \mathbf{H}_{c}^{i}\right)= \begin{cases}\pi(\theta)^{\oplus d}, & \text { si } i=d-1 ; \\ 0, & \text { sinon. }\end{cases}
$$

Preuve: Notons $M(\theta)$ la partie $\theta$-isotypique d'un $\mathbb{F}_{q^{d}}^{\times}$-module $M$. Rappelons que si $s$ est un sommet de $\mathcal{B} \mathcal{T}$, nous avons pour tout $i \in \mathbb{N}$ un isomorphisme (cf. (2.5.6))

$$
H_{c}^{i}\left(\nu^{-1}\left(|s|^{*}\right), \overline{\mathbb{Q}}_{\ell}\right) \cong H_{c}^{i}\left(\mathrm{DL}_{\overline{\mathbb{F}}_{q}}^{d-1}, \overline{\mathbb{Q}}_{\ell}\right)
$$

Comme $\theta$ est $d$-primitif, grâce à la propriété des variétés de Deligne-Lusztig [DL76, Lemma $9.14]$, on a

$$
H_{c}^{i}\left(\mathrm{DL}_{\overline{\mathbb{F}}_{q}}^{d-1}, \overline{\mathbb{Q}}_{\ell}\right)(\theta) \cong \begin{cases}\bar{\pi}_{\theta}, & \text { si } i=d-1 \\ 0, & \text { sinon. }\end{cases}
$$

En vertu du théorème $(2.5 .9)$, si $i \neq d-1, H_{c}^{i}\left(\nu^{-1}\left(|\sigma|^{*}\right), \overline{\mathbb{Q}}_{\ell}\right)(\theta)=0 \forall \sigma$. Si $i=d-1, \sigma \ni s$ un simplexe tel que $\operatorname{dim} \sigma>0$, comme $\bar{\pi}_{\theta}$ est une représentation cuspidale de $\mathrm{GL}_{d}\left(\mathbb{F}_{q}\right)$, on a

$$
H_{c}^{d-1}\left(\nu^{-1}\left(|\sigma|^{*}\right), \overline{\mathbb{Q}}_{\ell}\right)(\theta) \cong \bar{\pi}_{\theta}^{G_{\sigma}^{+} / G_{s}^{+}}=0
$$

Donc, la partie $\theta$-isotypique de la suite spectrale 3.1 .2 dégénère en $E_{1}$ lorsque $i=d-1$. En conclusion, on a

$$
\operatorname{Hom}_{\mathbb{F}_{q^{d}}^{\times}}\left(\theta, H_{c}^{i}\left(\Sigma^{c a}, \overline{\mathbb{Q}}_{\ell}\right)\right)= \begin{cases}\bigoplus_{s \in \mathcal{B} \mathcal{T}_{0}} H_{c}^{d-1}\left(\nu^{-1}\left(|s|^{*}\right), \overline{\mathbb{Q}}_{\ell}\right)(\theta)=\bigoplus_{s \in \mathcal{B} \mathcal{T}_{0}} \bar{\pi}_{\theta}, & \text { si } i=d-1 ; \\ 0, & \text { sinon. }\end{cases}
$$

Ceci nous fournit un isomorphisme de $\overline{\mathbb{Q}}_{\ell}[G]_{d}$-modules

$$
\left.\operatorname{Hom}_{\mathbb{F}_{q^{d}}^{\times}}\left(\theta, H_{c}^{d-1}\left(\Sigma^{c a}, \overline{\mathbb{Q}}_{\ell}\right)\right) \cong \pi(\theta)\right|_{[G]_{d}} .
$$

Alors, en tant que représentation de $G$, nous avons

$$
\begin{aligned}
\operatorname{Hom}_{D^{\times}}\left(\rho(\theta), \mathbf{H}_{c}^{d-1}\right) & =\operatorname{ind}_{[G]_{d}}^{G} \operatorname{Hom}_{\mathbb{F}_{q^{d}}^{\times}}\left(\theta, H_{c}^{d-1}\left(\Sigma^{c a}, \overline{\mathbb{Q}}_{\ell}\right)\right) \\
& =\left.\operatorname{ind}_{[G]_{d}}^{G} \pi(\theta)\right|_{[G]_{d}}
\end{aligned}
$$

Notons $\widetilde{\pi}(\theta):=\operatorname{ind}_{\mathrm{GL}_{d}(\mathcal{O}) \varpi^{\mathbb{Z}}}^{[G]_{d}} \bar{\pi}_{\theta}$, il s'ensuit que

$$
\left.\operatorname{ind}_{[G]_{d}}^{G} \pi(\theta)\right|_{[G]_{d}}=\operatorname{ind}_{[G]_{d}}^{G}\left(\bigoplus_{x \in G /[G]_{d}} x \widetilde{\pi}(\theta)\right)=\bigoplus_{x \in G /[G]_{d}} \operatorname{ind}_{[G]_{d}}^{G}\left({ }^{x} \widetilde{\pi}(\theta)\right),
$$


où ${ }^{x} \widetilde{\pi}(\theta)(g)=\widetilde{\pi}(\theta)\left(x^{-1} g x\right)$ pour $g \in[G]_{d}$. D'après la formule de Mackey, on sait que

$$
\operatorname{ind}_{[G]_{d}}^{G}\left({ }^{x} \tilde{\pi}(\theta)\right)=\pi(\theta), \forall x \in G /[G]_{d} .
$$

Ceci nous permet de conclure car $\left|G /[G]_{d}\right|=d$.

Considérons le $W_{K}$-module

$$
\operatorname{Hom}_{G}\left(\pi(\theta), \operatorname{Hom}_{D^{\times}}\left(\rho(\theta), \mathbf{H}_{c}^{d-1}\right)\right) .
$$

Dans le reste de ce paragraphe, on démontre la proposition suivante :

(3.1.10) Proposition.- On a un isomorphisme de $W_{K}$-modules

$$
\operatorname{Hom}_{G}\left(\pi(\theta), \operatorname{Hom}_{D^{\times}}\left(\rho(\theta), \mathbf{H}_{c}^{d-1}\right)\right) \cong \sigma^{\sharp}(\theta) .
$$

Preuve : D'après la réciprocité de Frobenius, on a

$$
\begin{aligned}
\operatorname{Hom}_{G}\left(\pi(\theta), \operatorname{Hom}_{D^{\times}}\left(\rho(\theta), \mathbf{H}_{c}^{d-1}\right)\right) & =\operatorname{Hom}_{G}\left(\operatorname{ind}_{\mathrm{GL}_{d}(\mathcal{O}) \varpi^{\mathbb{Z}}}^{G} \bar{\pi}_{\theta}, \operatorname{ind}_{[G W]_{d}}^{G W} \operatorname{Hom}_{\mathbb{F}_{q^{d}}^{\times}}\left(\theta, H_{c}^{d-1}\left(\Sigma^{c a}, \overline{\mathbb{Q}}_{\ell}\right)\right)\right. \\
& =\operatorname{ind}_{\left[W_{K}\right]_{d}}^{W_{K}} \operatorname{Hom}_{\mathrm{GL}_{d}(\mathcal{O}) \varpi^{\mathbb{Z}}}\left(\bar{\pi}_{\theta}, \operatorname{Hom}_{\mathbb{F}_{q^{d}}^{\times}}\left(\theta, H_{c}^{d-1}\left(\Sigma^{c a}, \overline{\mathbb{Q}}_{\ell}\right)\right)\right.
\end{aligned}
$$

D'après 3.1.9,

$$
\begin{aligned}
\operatorname{Hom}_{G}\left(\pi(\theta), \operatorname{Hom}_{D^{\times}}\left(\rho(\theta), \mathbf{H}_{c}^{d-1}\right)\right) & =\operatorname{ind}_{\left[W_{K}\right]_{d}}^{W_{K}} \operatorname{Hom}_{\mathrm{GL}_{d}(\mathcal{O}) \varpi^{\mathbb{Z}}}\left(\bar{\pi}_{\theta}, \bigoplus_{s^{\prime} \in \mathcal{B} \mathcal{T}_{0}} H_{c}^{d-1}\left(\nu^{-1}\left(\left|s^{\prime}\right|^{*}\right), \overline{\mathbb{Q}}_{\ell}\right)(\theta)\right) \\
& =\operatorname{ind}_{\left[W_{K}\right]_{d}}^{W_{K}} \operatorname{Hom}_{\mathrm{GL}_{d}\left(\mathbb{F}_{q}\right)}\left(\bar{\pi}_{\theta},\left(\bigoplus_{s^{\prime} \in \mathcal{B} \mathcal{T}_{0}} H_{c}^{d-1}\left(\nu^{-1}\left(\left|s^{\prime}\right|^{*}\right), \overline{\mathbb{Q}}_{\ell}\right)(\theta)\right)^{G_{s}^{+}}\right),
\end{aligned}
$$

où $s$ désigne le réseau standard $\left[\mathcal{O}^{d}\right]$. On démontre que

$$
\left(\bigoplus_{s^{\prime} \in \mathcal{B} \mathcal{T}_{0}} H_{c}^{d-1}\left(\nu^{-1}\left(\left|s^{\prime}\right|^{*}\right), \overline{\mathbb{Q}}_{\ell}\right)(\theta)\right)^{G_{s}^{+}}=H_{c}^{d-1}\left(\nu^{-1}\left(|s|^{*}\right), \overline{\mathbb{Q}}_{\ell}\right)(\theta)
$$

Évidemment, les éléments dans $H_{c}^{d-1}\left(\nu^{-1}\left(|s|^{*}\right), \overline{\mathbb{Q}}_{\ell}\right)(\theta)$ sont $G_{s}^{+}$-invariants. Soit $x=\sum x_{s^{\prime}} \in$ $\left(\bigoplus_{s^{\prime} \in \mathcal{B} \mathcal{T}_{0}} H_{c}^{d-1}\left(\nu^{-1}\left(\left|s^{\prime}\right|^{*}\right), \overline{\mathbb{Q}}_{\ell}\right)(\theta)\right)^{G_{s}^{+}}$avec $x_{s^{\prime}} \in H_{c}^{d-1}\left(\nu^{-1}\left(\left|s^{\prime}\right|^{*}\right), \overline{\mathbb{Q}}_{\ell}\right)(\theta)$. Notons $e_{G_{s^{\prime}}^{+}} l^{\prime}$ idempotent central associé à pro- $p$-groupe $G_{s^{\prime}}^{+}$. Par hypothèse, on a $e_{G_{s}^{+}} x=x$ et $e_{G_{s^{\prime}}^{+}} x_{s^{\prime}}=x_{s^{\prime}}, \forall s^{\prime}$. Soit $s^{\prime}$ un sommet différent de $s$, on a $e_{G_{s}^{+}} x_{s^{\prime}}=0$. En effet, il existe un sommet $s^{\prime \prime}$ dans l'enclos de $s$ et $s^{\prime}$ tel que $s^{\prime}, s^{\prime \prime}$ soient adjacent. D'après [MS10, 2.2], $e_{G_{s}^{+}} e_{G_{s^{\prime}}^{+}}=e_{G_{s}^{+}} e_{G_{s^{\prime \prime}}^{+}} e_{G_{s^{\prime}}^{+}}$. Notons que $G_{s^{\prime \prime}}^{+} G_{s^{\prime}}^{+} / G_{s^{\prime}}^{+}$est isomorphe à un sous-groupe unipotent non trivial de $\mathrm{GL}_{d}\left(\mathbb{F}_{q}\right)$, et que $H_{c}^{d-1}\left(\nu^{-1}\left(\left|s^{\prime}\right|^{*}\right), \overline{\mathbb{Q}}_{\ell}\right)(\theta)$ est cuspidale en tant que représentation de $G_{s^{\prime}} / G_{s^{\prime}}^{+} \cong \mathrm{GL}_{d}\left(\mathbb{F}_{q}\right)$. On a alors

$$
e_{G_{s}^{+}} x_{s^{\prime}}=e_{G_{s}^{+}} e_{G_{s^{\prime}}^{+}} x_{s^{\prime}}=e_{G_{s}^{+}}\left(e_{G_{s^{\prime \prime}}^{+}} e_{G_{s^{\prime}}^{+}} x\right)=0 .
$$

On en déduit que

$$
x=e_{G_{s}^{+}} x=e_{G_{s}^{+}} x_{s}+\sum_{s^{\prime} \neq s} e_{G_{s}^{+}} x_{s^{\prime}}=x_{s} \in H_{c}^{d-1}\left(\nu^{-1}\left(|s|^{*}\right), \overline{\mathbb{Q}}_{\ell}\right)(\theta) .
$$


Par conséquent, on a

$$
\operatorname{Hom}_{G}\left(\pi(\theta), \operatorname{Hom}_{D^{\times}}\left(\rho(\theta), \mathbf{H}_{c}^{d-1}\right)\right)=\operatorname{ind}_{\left[W_{K}\right]_{d}}^{W_{K}} \operatorname{Hom}_{\mathrm{GL}_{d}\left(\mathbb{F}_{q}\right)}\left(\bar{\pi}_{\theta}, H_{c}^{d-1}\left(\nu^{-1}\left(|s|^{*}\right), \overline{\mathbb{Q}}_{\ell}\right)(\theta)\right) .
$$

Notons que

$$
V:=\operatorname{Hom}_{\mathrm{GL}_{d}\left(\mathbb{F}_{q}\right)}\left(\bar{\pi}_{\theta}, H_{c}^{d-1}\left(\nu^{-1}\left(|s|^{*}\right), \overline{\mathbb{Q}}_{\ell}\right)(\theta)\right)
$$

est un $\overline{\mathbb{Q}}_{\ell^{-}}$espace vectoriel de dimension 1 , car

$$
H_{c}^{d-1}\left(\nu^{-1}\left(|s|^{*}\right), \overline{\mathbb{Q}}_{\ell}\right)(\theta) \cong H_{c}^{d-1}\left(\mathrm{DL}_{\bar{F}_{q}}, \overline{\mathbb{Q}}_{\ell}\right)(\theta)=\bar{\pi}_{\theta}
$$

en tant que représentation de $\mathrm{GL}_{d}\left(\mathbb{F}_{q}\right)$. Donc on se ramène à comprendre l'action de $\left[W_{K}\right]_{d}=$ $I_{K}\left\langle\varphi^{d}\right\rangle^{\mathbb{Z}}$ sur $V$.

Tout d'abord, on note que la dualité de Poincaré entraîne que

$$
\begin{aligned}
V & =\operatorname{Hom}_{\mathrm{GL}_{d}\left(\mathbb{F}_{q}\right)}\left(\bar{\pi}_{\theta}, H^{d-1}\left(\nu^{-1}(|s|), \overline{\mathbb{Q}}_{\ell}(d-1)\right)\left(\theta^{-1}\right)^{\vee}\right) . \\
& =\operatorname{Hom}_{\mathrm{GL}_{d}\left(\mathbb{F}_{q}\right)}\left(\bar{\pi}_{\theta}, H^{d-1}\left(\Sigma_{s}^{c a}, \overline{\mathbb{Q}}_{\ell}(d-1)\right)\left(\theta^{-1}\right)^{\vee}\right) .
\end{aligned}
$$

En comparant (2.3.8) et (2.5.1), on observe que l'action de $I_{K}$ sur $\widehat{\Sigma}_{s}^{0}(c f .(2.3 .8))$ se factorise par $I_{K} / I_{K\left(\varpi_{t}\right)} \cong \mathbb{F}_{q^{d}}^{\times}$, et elle s'identifie à l'action de $\mathbb{F}_{q^{d}}^{\times}$sur $\mathrm{DL}_{\overline{\mathbb{F}}_{q}}^{d-1}$. Alors l'action de $I_{K}$ sur $V$ se factorise par $I_{K} / I_{K\left(\varpi_{t}\right)}$ via le caractère $\theta$.

On est amené donc à étudier l'action de $\varphi^{d}$ sur $V$. À priori, l'espace analytique $\Sigma^{c a}$ n'a qu'une action du groupe d'inertie $I_{K}$. Cependant la donnée de descente à la Weil sur $\widehat{\mathcal{M}}_{D r, 0}$ ([RZ96, (3.48)]) définit une action de l'élément de Frobenius géométrique $\varphi$ sur $\mathcal{M}_{D r, 1}^{c a}$. Pour définir une donnée de descente de $\Sigma$, il faut décaler par l'endomorphisme $\Pi_{D}^{-1}$. Précisément, d'après [RZ96, (3.72)], le morphisme $\Pi_{D}^{-1} \circ \varphi$ induit la donnée de descente canonique de $\widehat{\mathcal{M}}_{D r, 0}^{(0)}$, donc il induit une donnée de descente de $\Sigma$, et de même une donnée de descente de $\widehat{\Sigma}_{s}^{0}$. En particulier, $\Pi_{D}^{-1} \circ \varphi$ induit une donnée de descente de la fibre spéciale $\bar{\Sigma}_{s}^{0}$ de $\widehat{\Sigma}_{s}^{0}$. Autrement dit, cela nous fournit une structure $\mathbb{F}_{q}$-rationnelle de $\bar{\Sigma}_{s}^{0}$, et on note Fr le morphisme de Frobenius associé.

Pour déterminer cette structure $\mathbb{F}_{q}$-rationnelle, on considère l'action de Fr sur les composantes connexes géométriques de $\bar{\Sigma}_{s}^{0}$. Notons $\pi_{0}(X)$ l'ensemble des composantes connexes géométriques de $X$ pour un espace analytique ou une variété $X$. On a le fait suivant:

FAIT.- $\pi_{0}\left(\mathcal{M}_{D r, 1}\right)$ est un espace homogène sous $K^{\times}$, isomorphe à $K^{\times} /(1+\varpi \mathcal{O})$, sur lequel l'action de $G$ est donnée par $G \stackrel{\text { det }}{\longrightarrow} K^{\times}$, celle de $D^{\times}$est donnée par $D^{\times} \stackrel{\text { Nr }}{\longrightarrow} K^{\times}$, celle de $W_{K}$ est donnée par $\operatorname{Art}_{K}^{-1}: W_{K} \rightarrow W_{K}^{a b} \stackrel{\sim}{\longrightarrow} K^{\times}$.

Remarque.- Dans [Che13], Chen a étudié les composantes connexes géométriques de la tour de l'espace de Rapoport-Zink non-ramifié de certains types, avec les actions de différents groupes associés à la donnée de Rapoport-Zink. En particulier, elle a décrit les composantes connexes géométriques de la tour de Lubin-Tate $\left(\mathcal{M}_{L T, n}\right)_{n \in \mathbb{N}}$ munies des actions de $G, D^{\times}, W_{K}$. En vertu de l'isomorphisme de Faltings-Fargues [Far08] entre la tour de Lubin-Tate et la tour de Drinfeld et en prenant le sous-ensemble fixé par $1+\Pi_{D} \mathcal{O}_{D}$, on obtient l'énoncé du fait précédent.

LEMME.- $\pi_{0}\left(\bar{\Sigma}_{s}^{0}\right)$ est un espace principal homogène sous $\mathbb{F}_{q}^{\times}$sur lequel Fr agit par multiplication par $(-1)^{d-1}$. 
Preuve: Notons que $\operatorname{Nr}\left(\Pi_{D}\right)=(-1)^{d-1} \varpi$ et $\operatorname{Art}^{-1}(\varphi)=\varpi$. D'après le fait précédent, $\pi_{0}(\Sigma)$ est un espace principal homogène sous $\mathbb{F}_{q}^{\times}$, sur lequel $\Pi_{D}^{-1} \circ \varphi$ agit par multiplication par

$$
\operatorname{Nr}\left(\Pi_{D}^{-1}\right) \cdot \operatorname{Art}^{-1}(\varphi)=(-1)^{d-1} \varpi^{-1} \cdot \varpi=(-1)^{d-1} .
$$

Comme $\widehat{\Sigma}_{s}^{0}$ est un $\mu_{q^{d}-1}$-torseur sur $\widehat{\Omega}_{\breve{\mathcal{O}}, s}^{d-1}$, on a une bijection canonique entre $\pi_{0}\left(\nu^{-1}(|s|)\right)$ et $\pi_{0}\left(\bar{\Sigma}_{s}^{0}\right)$. D'après l'équation 2.5.2 de $\bar{\Sigma}_{s}^{0} \cong \mathrm{DL}_{\overline{\mathbb{F}}_{q}}^{d-1}$, on sait que $\left|\pi_{0}\left(\bar{\Sigma}_{s}^{0}\right)\right|=q-1$. Les immersions $\nu^{-1}(|s|) \rightarrow \nu^{-1}\left(|s|^{*}\right) \rightarrow \Sigma$ induisent les morphismes $\alpha, \beta$ entre les composantes connexes

$$
\pi_{0}\left(\nu^{-1}(|s|)\right) \stackrel{\alpha}{\longrightarrow} \pi_{0}\left(\nu^{-1}\left(|s|^{*}\right)\right) \stackrel{\beta}{\longrightarrow} \pi_{0}(\Sigma) .
$$

On démontre que le composé $\beta \circ \alpha$ est une bijection. En effet, $\alpha$ est bijective, car $H^{0}\left(\nu^{-1}(|s|), \Lambda\right)=$ $H^{0}\left(\nu^{-1}\left(|s|^{*}\right), \Lambda\right)$, d'après (2.2.3). Puisque $\left|\pi_{0}\left(\nu^{-1}(|s|)\right)\right|=\left|\pi_{0}(\Sigma)\right|=q-1$, il suffit de montrer que $\beta$ est surjective. Notons que $\left\{\nu^{-1}\left(\left|s^{\prime}\right|^{*}\right)\right\}_{s^{\prime} \in \mathcal{B} \mathcal{T}_{0}}$ forment un recourement ouvert de $\Sigma$. On se ramène alors à prouver que pour $s^{\prime}$ un sommet quelconque et $U^{\prime}$ une composante connexe de $\pi_{0}\left(\nu^{-1}\left(|s|^{*}\right)\right)$, il existe une suite de sommets $s_{0}=s, s_{1}, \ldots, s_{n}=s^{\prime}$ et des composantes conexes $U_{i} \in \pi_{0}\left(\nu^{-1}\left(\left|s_{i}\right|^{*}\right)\right)$ avec $U_{n}=U^{\prime}$ telles que $s_{i}, s_{i+1}$ soient adjacent et $U_{i} \cap U_{i+1} \neq \emptyset, \forall i$. Par dévissage, il suffit de considérer le cas où $s$ et $s^{\prime}$ sont adjacent. Notons $\sigma$ le simplexe $\left[s, s^{\prime}\right]$, et considérons les immersions ouvertes $\nu^{-1}\left(|\sigma|^{*}\right) \hookrightarrow \nu^{-1}\left(|s|^{*}\right)$ et $\nu^{-1}\left(|\sigma|^{*}\right) \hookrightarrow \nu^{-1}\left(\left|s^{\prime}\right|^{*}\right)$ induisant les morphismes entre $\pi_{0}$ :

$$
\alpha_{1}: \pi_{0}\left(\nu^{-1}\left(|\sigma|^{*}\right)\right) \rightarrow \pi_{0}\left(\nu^{-1}\left(|s|^{*}\right)\right), \alpha_{2}: \pi_{0}\left(\nu^{-1}\left(|\sigma|^{*}\right)\right) \rightarrow \pi_{0}\left(\nu^{-1}\left(\left|s^{\prime}\right|^{*}\right)\right) .
$$

Notons que $\alpha_{1}$ et $\alpha_{2}$ sont bijectives. Ceci découle du théorème (2.5.9) et du fait que le $p$ groupe $G_{\sigma}^{+}$agit trivialement sur $H_{c}^{2 d-2}\left(\nu^{-1}\left(|s|^{*}\right), \Lambda\right)$. Donc, pour $U^{\prime} \in \pi_{0}\left(\nu^{-1}\left(\left|s^{\prime}\right|^{*}\right)\right)$, il existe une composante connexe $U \in \pi_{0}\left(\nu^{-1}\left(|s|^{*}\right)\right)$ tel que $U \cap U^{\prime}$ contienne une composante connexe du ouvert $\nu^{-1}\left(|\sigma|^{*}\right)$ de $\Sigma$. Par conséquent, $\beta$ est bijective.

On a alors une bijection canonique entre $\pi_{0}(\Sigma)$ et $\pi_{0}\left(\bar{\Sigma}_{s}^{0}\right)$ compatible avec l'action de $\mathbb{F}_{q}^{\times}$. Par conséquent, le morphisme de Frobenius Fr induit la multiplication par $(-1)^{d-1} \operatorname{sur} \pi_{0}\left(\bar{\Sigma}_{s}^{0}\right)$.

(3.1.11) LeMme.- La forme $\mathbb{F}_{q}$-rationnelle de $\bar{\Sigma}_{s}^{0}$ induite par la donnée de descente Fr est isomorphe à la variété de Deligne-Lusztig $\left(\mathbb{F}_{q}\right.$-rationnelle) $\mathrm{DL}^{d-1}$ définie par l'équation 2.5.2.

Preuve : D'après le théorème (2.5.4), on sait que $\bar{\Sigma}_{s}^{0} \cong \mathrm{DL}_{\overline{\mathbb{F}}_{q}}^{d-1}$. Grâce à la suite spectrale

$$
E_{2}^{p q}=H^{p}\left(\operatorname{Gal}\left(\overline{\mathbb{F}}_{q} / \mathbb{F}_{q}\right), H^{q}\left(\Omega_{\overline{\mathbb{F}}_{q}}^{d-1}, \mu_{q^{d}-1}\right)\right) \Longrightarrow H^{p+q}\left(\Omega_{\mathbb{F}_{q}}^{d-1}, \mu_{q^{d}-1}\right)
$$

on a une suite exacte

$0 \rightarrow H^{1}\left(\operatorname{Gal}\left(\overline{\mathbb{F}}_{q} / \mathbb{F}_{q}\right), \mu_{q^{d}-1}\right) \rightarrow H^{1}\left(\Omega_{\mathbb{F}_{q}}^{d-1}, \mu_{q^{d}-1}\right) \rightarrow H^{1}\left(\Omega_{\overline{\mathbb{F}}_{q}}^{d-1}, \mu_{q^{d}-1}\right) \rightarrow H^{2}\left(\operatorname{Gal}\left(\overline{\mathbb{F}}_{q} / \mathbb{F}_{q}\right), \mu_{q^{d}-1}\right)$

Notons que $H^{1}\left(\operatorname{Gal}\left(\overline{\mathbb{F}}_{q} / \mathbb{F}_{q}\right), \mu_{q^{d}-1}\right)=\mathbb{F}_{q}^{\times}$grâce à la suite exacte de Kummer et Thm. 90 de Hilbert, et que $H^{2}\left(\operatorname{Gal}\left(\overline{\mathbb{F}}_{q} / \mathbb{F}_{q}\right), \mu_{q^{d}-1}\right)=0$. On en déduit que les formes $\mathbb{F}_{q^{-}}$rationnelles de $\mathrm{DL}_{\overline{\mathbb{F}}_{q}}$ sont données par $Y_{a}$ pour tout $a \in \mathbb{F}_{q}^{\times}$, où $Y_{a}$ est donnée par l'équation $\operatorname{det}\left(\left(X_{i}^{q^{j}}\right)_{0 \leqslant i, j \leqslant d-1}\right)^{q-1}=$ a. En particulier, $\mathrm{DL}^{d-1}=Y_{(-1)}{ }^{d-1}$.

L'ensemble des composantes connexes géométriques $\pi_{0}\left(Y_{a}\right)$ de $Y_{a}$ s'identifie à l'ensemble des racines $(q-1)$-ièmes de $a$. Précisément, on associe $\varepsilon$ telle que $\varepsilon^{q-1}=a$ la composante 
connexe géométrique définie par l'équation $\operatorname{det}\left(\left(X_{i}^{q^{j}}\right)_{0 \leqslant i, j \leqslant d-1}\right)=\varepsilon$. Via cette identification, $t \in \mathbb{F}_{q}^{\times}$agit par multiplication qui envoie $\varepsilon$ vers $t \varepsilon$. Considérons le morphisme de Frobenius Frob : $\left(X_{i}\right)_{0 \leqslant i \leqslant d-1} \mapsto\left(X_{i}^{q}\right)_{0 \leqslant i \leqslant d-1}$ sur $Y_{a}$. Pour un point $x=\left(x_{0}, \ldots, x_{d-1}\right)$ tel que $\operatorname{det}\left(\left(x_{i}^{q^{j}}\right)_{0 \leqslant i, j \leqslant d-1}\right)=\varepsilon, \operatorname{Frob}(x)=\left(x_{0}^{q}, \ldots, x_{d-1}^{q}\right)$ satisfait l'équation $\operatorname{det}\left(\left(x_{i}^{q}\right)^{q^{j}}\right)_{0 \leqslant i, j \leqslant d-1}=$ $\varepsilon^{q}=a \cdot \varepsilon$. Autrement dit, Frob agit sur $\pi_{0}\left(Y_{a}\right)$ par multiplication par $a$. Il s'ensuit que $Y_{(-1)^{d-1}}=\mathrm{DL}^{d-1}$ est la forme $\mathbb{F}_{q^{-}}$rationnelle de $\bar{\Sigma}_{s}^{0}$ induite par Fr, en vertu du lemme précédent.

(3.1.12) ThÉORÈme.- (Digne et Michel [DM85]) Fr $^{d}$ agit sur $H_{c}^{d-1}\left(\bar{\Sigma}_{s}^{0}, \overline{\mathbb{Q}}_{\ell}\right)(\theta)$ par multiplication par $(-1)^{d-1} q^{\frac{d(d-1)}{2}}$.

Preuve : D'après le lemme précédent, il suffit de montrer que $(-1)^{d-1} q^{\frac{d(d-1)}{2}}$ est l'unique valeur propre de Frob $^{d}$ sur $H_{c}^{d-1}\left(\mathrm{DL}^{d-1}, \overline{\mathbb{Q}}_{\ell}\right)(\theta)$. Ceci est essentiellement démontré par Digne et Michel [DM85, V. (3.14)]. Cependant, ils ont calculé la valeur propre de Frob $^{d}$ pour la variété définie par l'équation $\operatorname{det}\left(\left(X_{i}^{q^{j}}\right)_{0 \leqslant i, j \leqslant d-1}\right)^{q-1}=1$. De plus, dans leur formule (page 106 de loc. cit.), il manque un signe $(-1)^{d-1}(d=n$ dans loc. cit.), comme ils ont oublié qu'ils travaillaient avec une somme alternée. En plus, le facteur $\theta\left((-1)^{d-1}\right)$ dans loc. cit. disparait. Cela vient du fait que l'on peut rendre un isomorphisme entre $Y_{1}$ et $Y_{a}$ par un changement de variables $f: X_{i} \mapsto X_{i} / b$ où $b^{q^{d}-1}=a$. Alors l'action de Frob ${ }^{d}$ sur $H_{c}^{i}\left(Y_{a}, \overline{\mathbb{Q}}_{\ell}\right)$ est la même que celle sur $H_{c}^{i}\left(Y_{1}, \overline{\mathbb{Q}}_{\ell}\right)$ tordue par l'automorphisme induit par le changement de variables $X_{i} \mapsto a \cdot X_{i}$, donc n'est autre que l'action de l'élément $a \in \mathbb{F}_{q^{d}}^{\times}$. Ce dernier agit bien par $\theta(a)$ sur la partie $\theta$-isotypique de la cohomologie. Par conséquent, dans notre cas où $a=(-1)^{d-1}$, l'endomorphisme Frob ${ }^{d}$ agit sur $H_{c}^{d-1}\left(\mathrm{DL}^{d-1}, \overline{\mathbb{Q}}_{\ell}\right)(\theta)$ par la constante $(-1)^{d-1} q^{\frac{d(d-1)}{2}}$.

Revenons à la preuve de la proposition (3.1.10). L'action de $\varphi^{d}$ sur $V$ est donnée par celle sur $H^{d-1}\left(\Sigma_{s}^{c a}, \overline{\mathbb{Q}}_{\ell}(d-1)\right)(\theta)^{\vee}$, donc elle s'identifie à l'action de $\operatorname{Fr}^{d}$ sur $H_{c}^{d-1}\left(\bar{\Sigma}_{s}^{0}, \overline{\mathbb{Q}}_{\ell}\right)(\theta)$. En vertu du lemme précédent, on obtient l'énoncé de la proposition.

\section{Références}

[BC91] J.-F. Boutot et H. CARAyol : Uniformisation $p$-adique des courbes de Shimura : les théorèmes de Čerednik et de Drinfel'd. Astérisque, (196-197):7, 45-158 (1992), 1991. Courbes modulaires et courbes de Shimura (Orsay, 1987/1988).

[Ber93] Vladimir G. Berkovich : Étale cohomology for non-Archimedean analytic spaces. Publ. Math. Inst. Hautes Études Sci., 78:5-161, 1993.

[Ber95] Vladimir G. Berkovich : The automorphism group of the Drinfel'd half-plane. C. R. Acad. Sci. Paris Sér. I Math., 321(9):1127-1132, 1995.

[Ber96] Vladimir G. Berkovich : Vanishing cycles for formal schemes. II. Invent. Math., 125(2):367-390, 1996.

[Boy99] Pascal Boyer : Mauvaise réduction des variétés de Drinfeld et correspondance de Langlands locale. Invent. Math., 138(3):573-629, 1999.

[Car90] H. Carayol : Nonabelian Lubin-Tate theory. In Automorphic forms, Shimura varieties, and L-functions, Vol. II (Ann Arbor, MI, 1988), volume 11 de Perspect. Math., pages 15-39. Academic Press, Boston, MA, 1990. 
[Che13] Miaofen Chen : Composantes connexes géométriques de la tour des espaces de modules de groupes p-divisibles. À paraître dans Ann. Sci. École Norm. Sup., 2013.

[Dat06] Jean-François DAT : Espaces symétriques de Drinfeld et correspondance de Langlands locale. Ann. Sci. École Norm. Sup. (4), 39(1):1-74, 2006.

[Dat07] Jean-François DAT : Théorie de Lubin-Tate non-abélienne et représentations elliptiques. Invent. Math., 169(1):75-152, 2007.

[DH87] Pierre Deligne et Dale Husemoller : Survey of Drinfel'd modules. In Current trends in arithmetical algebraic geometry (Arcata, Calif., 1985), volume 67 de Contemp. Math., pages 25-91. Amer. Math. Soc., Providence, RI, 1987.

[DL76] P. Deligne et G. Lusztig : Representations of reductive groups over finite fields. Ann. of Math. (2), 103(1):103-161, 1976.

[DM85] François Digne et Jean Michel : Fonctions $L$ des variétés de Deligne-Lusztig et descente de Shintani. Mém. Soc. Math. France (N.S.), (20):iv+144, 1985.

[Dri74] V. G. DrinfeL'D : Elliptic modules. Mat. Sb. (N.S.), 94(136):594-627, 656, 1974.

[Dri76] V. G. Drinfel'D : Coverings of p-adic symmetric domains. Funkcional. Anal. $i$ Priložen., 10(2):29-40, 1976.

[Far08] Laurent FArgues : L'isomorphisme entre les tours de Lubin-Tate et de Drinfeld et applications cohomologiques. In L'isomorphisme entre les tours de Lubin-Tate et de Drinfeld, volume 262 de Progr. Math., pages 1-325. Birkhäuser, Basel, 2008.

[FvdP04] Jean Fresnel et Marius van der PUT : Rigid analytic geometry and its applications, volume 218 de Progress in Mathematics. Birkhäuser Boston Inc., Boston, MA, 2004.

[Gen] Alain Genestier : Exposés à Göttingen et à Harvard.

[Gen96] Alain Genestier : Espaces symétriques de Drinfeld. Astérisque, (234):ii+124, 1996.

[GI63] O. Goldman et N. Iwahori : The space of p-adic norms. Acta Math. 109, pages 137-177, 1963.

[Har77] Robin Hartshorne : Algebraic geometry. Springer-Verlag, New York, 1977. Graduate Texts in Mathematics, No. 52.

[Har97] Michael HARRIS : Supercuspidal representations in the cohomology of Drinfel'd upper half spaces ; elaboration of Carayol's program. Invent. Math., 129(1):75-119, 1997.

[Hau05] Thomas HaUsberger : Uniformisation des variétés de Laumon-Rapoport-Stuhler et conjecture de Drinfeld-Carayol. Ann. Inst. Fourier (Grenoble), 55(4):1285-1371, 2005 .

[HT01] Michael HARRIS et Richard TAYLOR : The geometry and cohomology of some simple Shimura varieties, volume 151 de Annals of Mathematics Studies. Princeton University Press, Princeton, NJ, 2001.

[Ito05] Tetsushi ITO : Weight-monodromy conjecture for $p$-adically uniformized varieties. Invent. Math., 159(3):607-656, 2005.

[Kur80] Akira Kurihara : Construction of $p$-adic unit balls and the Hirzebruch proportionality. Amer. J. Math., 102(3):565-648, 1980.

[MS10] Ralf MEYER et Maarten Solleveld : Resolutions for representations of reductive p-adic groups via their buildings. J. Reine Angew. Math., 647:115-150, 2010. 
[Mus78] G. A. Mustafin : Non-Archimedean uniformization. Mat. Sb. (N.S.), 105(147)(2): 207-237, 287, 1978.

[Rap90] M. RApoporT : On the bad reduction of Shimura varieties. In Automorphic forms, Shimura varieties, and L-functions, Vol. II (Ann Arbor, MI, 1988), volume 11 de Perspect. Math., pages 253-321. Academic Press, Boston, MA, 1990.

[Ray74] Michel Raynaud : Schémas en groupes de type $(p, \ldots, p)$. Bull. Soc. Math. France, 102:241-280, 1974.

[RZ96] M. RAPOPORT et Th. ZINK : Period spaces for p-divisible groups, volume 141 de Annals of Mathematics Studies. Princeton University Press, Princeton, NJ, 1996.

[SGA1] Revêtements étales et groupe fondamental. Lecture Notes in Mathematics, Vol. 224. Springer-Verlag, Berlin, 1971. Séminaire de géométrie algébrique du Bois Marie 1960-61.

[SGA7-1] Groupes de monodromie en géométrie algébrique. I. Lecture Notes in Mathematics, Vol. 288. Springer-Verlag, Berlin, 1972. Séminaire de Géométrie Algébrique du Bois-Marie 1967-1969 .

[SGA4-3] Théorie des topos et cohomologie étale des schémas. Tome 3. Lecture Notes in Mathematics, Vol. 305. Springer-Verlag, Berlin, 1973. Séminaire de Géométrie Algébrique du Bois-Marie 1963-1964 .

[SS93] P. Schneider et U. Stuhler : Resolutions for smooth representations of the general linear group over a local field. J. Reine Angew. Math., 436:19-32, 1993.

[Str08] Matthias STRAuch : Deformation spaces of one-dimensional formal modules and their cohomology. Adv. Math., 217(3):889-951, 2008.

[Tei90] Jeremy Teitelbaum : Geometry of an étale covering of the $p$-adic upper half plane. Ann. Inst. Fourier (Grenoble), 40(1):68-78, 1990.

[Wan13] Haoran WANG : Sur la cohomologie des compactifications de variétés de DeligneLusztig. À paraître dans Ann. Inst. Fourier (Grenoble), arXiv :1310.7259, 2013.

[Wan14] Haoran WANG : L'espace symétrique de Drinfeld et correspondance de Langlands locale II, arXiv :1402.1965, 2014.

[Yos10] Teruyoshi Yoshida : On non-abelian Lubin-Tate theory via vanishing cycles. In Algebraic and arithmetic structures of moduli spaces (Sapporo 2007), volume 58 de Adv. Stud. Pure Math., pages 361-402. Math. Soc. Japan, Tokyo, 2010.

[Zhe08] Weizhe Zheng: Sur la cohomologie des faisceaux $l$-adiques entiers sur les corps locaux. Bull. Soc. Math. France, 136(3):465-503, 2008.

HAORAN WANG

Université Pierre et Marie Curie, Institut de Mathématiques de Jussieu

Adresse Présente : Max-Planck-Institut für Mathematik, Vivatsgasse 7, 53111 Bonn, Germany haoran.wang@mpim-bonn.mpg.de 